\title{
Poisson measures for topological groups and their representations.
}

\author{
S.V. Ludkovsky \\ 01 December 1998 *
}

\section{Introduction}

In articles 17, 18, 19, 23, 24, 25, 27, 29, 30, 35 Gaussian quasi-invariant measures on groups of diffeomorphisms and loop groups $G$ relative to dense subgroups $G^{\prime}$ were constructed. In the non-Archimedean case the wider class of measures was investigated, than in the real case. The cases of Riemann and non-Archimedean manifolds were considered. There are few approaches for the construction of irreducible unitary representations. In articles 14, 18, 23, 24, 25 representations of dense subgroups $G^{\prime}$ associated with quasi-invariant measures on the entire groups were considered. In articles [12, 20, 21] irreducible representations of groups of diffeomorphisms $\operatorname{Diff}(\bar{M})$ associated with measures on specific subsets of the unital type of products $M^{\mathbf{N}}$ of the manifolds $M$ were investigated. In the publications [36, 38] irreducible unitary representations of groups of diffeomorphisms associated with real-valued Poisson measures on products of real manifolds were studied.

This article is related with unitary representations of $G^{\prime}$ associated with Poisson measures on $G^{\mathbf{N}}$ and uses quasi-invariant measures on $G$ from the previous works. Several groups are considered: (1) (a) diffeomorphisms and (b) loop groups of real manifolds, (2) (a) diffeomorphisms and (b) loop groups of non-Archimedean manifolds over local fields. Besides these four cases

*Mathematics subject classification (1991 Revision) 22A10, 43A05 and 46B. 
further the fifth and the sixth cases are considered: for (3) (a) real and (b) non-Archimedean groups of diffeomorphisms Diff $(M)$ representations associated with Poisson measures on configuration spaces $\Gamma_{M}$ contained in products of manifolds $M^{\mathbf{N}}$ are investigated. The case (3) (a) for real locally compact $M$ was considered in [36, 38]. Here the cases of infinite-dimensional Banach manifold $M(3)$ (a), non-Archimdean locally compact and non-locally compact Banach manifolds (3) (b) are investigated. For this quasi-invariant measures on $M$ relative to $\operatorname{Diff}(M)$ from [20, 21] are considered. Henceforth real-valued measures are considered. In $§ 2$ necessary Poisson measures are considered, definitions and notations are given. In $\S 3$ irreducible unitary representations are considered. Certainly not all results from [36, 38] can be transferred onto the cases considered here, moreover, there were necessary strong changes in many definitions, proofs and formulations of the theorems.

It is necessary to note that the theory of representations of non-locally compact groups differ substantially from that of locally compact groups. For example, irreducible unitary representations of locally compact Abelian groups are one-dimensional, that is, characters. But for non-locally compact Abelian groups there are infinite-dimensional irreducible unitary representations, which are even regular representations. It was shown in [1, 10] that there are infinite-dimensional topological vector spaces $E$ and dense nuclear additive subgroups $E^{\prime}$ such that $E^{\prime}$ are linear subspaces and quasi-invariant measures $\mu$ on $E$ relative to $E^{\prime}$ exist such that associated with them regular representations in the Hilbert space $L^{2}(E, \mu, \mathbf{C})$ are irreducible. The existence of such irreducible representations is even despite of the fact that projections $\mu_{J}$ of $\mu$ on one-dimensional subspaces $J$ are equivalent with the Haar measures on $J$. This shows that non-locally compact case is more complicated than it may be supposed at the first glance. Also for definite groups $G$ of diffeomorphisms and loops of definite real and non-Archimedean manifolds there are quasi-invariant measures $\mu$ on $G$ relative to dense subgroups $G^{\prime}$ such that associated with them regular unitary representations are irreducible [14, 18, 23, 24, 25]. Such difference is caused by the existence of $C^{*}$-algebras associated with the Haar measures on locally compact groups [11], but no any $C^{*}$-algebra can be directly associated with a non-zero quasi-invariant measure on a non-locally compact group relative to a dense subgroup $G^{\prime}$. Certainly, results on irreducibility of regular representations of infinite-dimensional topological groups $G^{\prime}$ depend stronlgy on quasi-invariant measures $\mu$ on $G$ and a structure of $G$, where $G^{\prime}$ is dense in $G$. 


\section{Poisson measures.}

2.1. Note. Let $X$ denotes a manifold $M$ for a group of diffeomorphisms $G=G(M)$ or the group $G$ itself, where $M$ is the $C^{\infty}$-manifold over $\mathbf{R}$ or an analytic manifold over a local field and $G$ is the loop group or the diffeomorphisms group as in the cited in $\S 1$ papers. Classes of smoothness of the groups $G$ and $G^{\prime}$ are considered to be not less than $C^{1}$. The groups of diffeomorphisms $G$ for the real $C^{\infty}$-manifold $M$ are denoted $D$ if $f_{\beta, \gamma}^{t}(M)$ with $\infty \geq t \geq 1, \beta \geq 0, \gamma \geq 0$; the loop groups $G$ for the real $C^{\infty}$-manifold $N$ are denoted $\left(L^{m} N\right)_{\gamma, Y}$ with $m+5<\gamma \leq \infty$, also another classes of smoothness and non-Archimedean groups and manifolds were considered (see theorem 3.4 [27 and also [6, 18, 23, 24, 21]). It was proved earlier, that $G$ itself is the $C^{\infty}$-manifold (in the case of the real group of diffeomorphisms for finite-dimensional Riemann manifolds $M$ see also [2, 6]). Moreover, in the non-Archimedean case $M$ and $G$ have structures of the analytic manifolds with clopen disjoint charts. Clearly, $G$ itself is not locally compact, since $G$ considered as the manifold is infinite-dimensional over the corresponding field. When $X=M$ let us suppose, that $X$ is the Banach non-compact manifold. In the non-Archimedean case it has embedding into the Banach space $Z$ over the same local field $\mathbf{L}$ due to the partition of $M$ into disjoint union of balls, so an atlas of $M$ is supposed to be analytic and it has automatically foliated structure [22, 26]. In the real case it is supposed that $M$ has a foliated structure with finite-dimensional submanifolds $M_{n} \subset M_{n+1}$ for each $n \in \mathbf{N}$ and $\bigcup_{n \in \mathbf{N}} M_{n}$ is dense in $M$, where $\operatorname{dim}_{\mathbf{R}} M_{n}=k_{n}<\infty$ [21, 27, 30].

We remind the definition of the configuration space from [36] and also consider the ultrametric case of $X$. This means that a metric $d$ in $X$ satisfies the ultrametric inequality $d(x, y) \leq \max (d(x, z), d(y, z))$ for each $x, y, z \in X$.

Let $K$ be a complete separable metric space with a metric $d$, that is, $X$ is a Polish space. In the ultrametric case this implies that its topological great inductive dimension is zero: $\operatorname{Ind}(K)=0$ [7]. Let $d_{K}^{n}(x, y):=\sum_{i=1}^{n} d\left(x_{i}, y_{i}\right)$ in the real case and $d(x, y):=\max _{1 \leq i \leq n} d\left(x_{i}, y_{i}\right)$ in the non-Archimedean case be a metric in $K^{n}$, where $x=\left(x_{i}: i=1, \ldots, n\right) \in K^{n}, x_{i} \in K$. Put $\tilde{K}^{n}:=\left(x \in K^{n}: x_{i} \neq x_{j}\right.$ for each $\left.i \neq j\right)$. Supply $\tilde{K}^{n}$ with a metric $\delta_{K}^{n}(x, y):=d_{K}^{n}(x, y) /\left[d_{K}^{n}(x, y)+d_{K}^{n}\left(x,\left(\tilde{K}^{n}\right)^{c}\right)+d_{K}^{n}\left(y,\left(\tilde{K}^{n}\right)^{c}\right)\right]$ in the real case and $\delta_{K}^{n}(x, y):=d_{K}^{n}(x, y) /\left[\max \left(d_{K}^{n}(x, y), d_{K}^{n}\left(x,\left(\tilde{K}^{n}\right)^{c}\right), d\left(y,\left(\tilde{K}^{n}\right)^{c}\right)\right]\right.$ in the non-Archimedean case, where $A^{c}:=K^{n} \backslash A$ for a subset $A \subset K^{n}$. Then $\left(\tilde{K}^{n}, \delta_{K}^{n}\right)$ is the Polish space. Moreover, if $(K, d)$ is ultrametric, then 
$\left(\tilde{K}^{n}, \delta_{K}^{n}\right)$ is ultrametric. Let also $B_{K}^{n}$ denotes the collection of all $n$-point subsets of $K$. Then the metric $\delta_{K}^{n}$ is equivalent with the following metric $d_{K}^{(n)}\left(\gamma, \gamma^{\prime}\right):=\inf _{\sigma \in \Sigma_{n}} d_{K}^{n}\left(\left(x_{1}, \ldots, x_{n}\right),\left(x_{\sigma(1)}^{\prime}, \ldots, x_{\sigma(n)}^{\prime}\right)\right)$, where $\Sigma_{n}$ is the symmetric group of $(1, \ldots, n), \sigma \in \Sigma_{n}, \sigma:(1, \ldots, n) \rightarrow(1, \ldots, n) ; \gamma, \gamma^{\prime} \in B_{K}^{n}$. For each subset $A \subset K$ a number mapping $N_{A}: B_{K}^{n} \rightarrow \mathbf{N}_{\mathbf{o}}$ is defined by the following formula: $N_{A}(\gamma):=\operatorname{card}(\gamma \cap A)$, where $\mathbf{N}:=\{1,2,3, \ldots\}$, $\mathbf{N}_{\mathbf{o}}:=\{0,1,2,3, \ldots\}$. Evidently, $d_{K}^{(n)}$ is the ultrametric, if $d_{K}^{n}$ is the ultrametric. It remains to show, that $\delta_{K}^{n}$ is the ultrametric for the ultrametric space $(K, d)$. For this we mention, that $(i) \delta_{K}^{n}(x, y)>0$, when $x \neq y$, and $\delta_{K}^{n}(x, x)=0$. (ii) $\delta_{K}^{n}(x, y)=\delta_{K}^{n}(y, x)$, since this symmetry is true for $d_{K}^{n}$ and for [*] in the denumerator in the formula defining $\delta_{K}^{n}$. To prove $(i i i) \delta_{K}^{n}(x, y) \leq \max \left(\delta_{K}^{n}(x, z), \delta_{K}^{n}(z, y)\right)$ we consider the case $\delta_{K}^{n}(x, z) \geq \delta_{K}^{n}(y, z)$, hence it is sufficient to show, that $\delta_{K}^{n}(x, y) \leq \delta_{K}^{n}(x, z)$. Let $(a) d_{K}^{n}(x, z) \geq \max \left(d_{K}^{n}\left(z,\left(\tilde{K}^{n}\right)^{c}\right), d_{K}^{n}\left(x,\left(\tilde{K}^{n}\right)^{c}\right)\right)$, then $\delta_{K}^{n}(x, z)=1$, hence $\delta_{K}^{n}(x, y) \leq \delta_{K}^{n}(x, z)$, since $\delta_{K}^{n}(x, y) \leq 1$ for each $x, y \in \tilde{K}^{n}$. Let $(b)$ $d_{K}^{n}\left(x,\left(\tilde{K}^{n}\right)^{c}\right)>\max \left(d_{K}^{n}(x, z), d_{K}^{n}\left(z,\left(\tilde{K}^{n}\right)^{c}\right)\right)$, then $\delta_{K}^{n}(x, z)=d_{K}^{n}(x, z) / d_{K}^{n}\left(x,\left(\tilde{K}^{n}\right)^{c}\right) \leq$ 1. Since $d_{K}^{n}(z, A):=\inf _{a \in A} d_{K}^{n}(z, a)$, then $d_{K}^{n}\left(z,\left(\tilde{K}^{n}\right)^{c}\right) \leq \max \left(d_{K}^{n}\left(y,\left(\tilde{K}^{n}\right)^{c}\right), d_{K}^{n}(y, z)\right)$. If $d_{K}^{n}(x, z)<d_{K}^{n}\left(z,\left(\tilde{K}^{n}\right)^{c}\right)$ and $d_{K}^{n}(x, y) \leq d_{K}^{n}(x, z)$, then $d_{K}^{n}\left(z,\left(\tilde{K}^{n}\right)^{c}\right) \leq$ $d_{K}^{n}\left(x,\left(\tilde{K}^{n}\right)^{c}\right)$. Hence $d_{K}^{n}(x, y) \max \left(d_{K}^{n}(x, z), d_{K}^{n}\left(x,\left(\tilde{K}^{n}\right)^{c}\right), d_{K}^{n}\left(z,\left(\tilde{K}^{n}\right)^{c}\right)\right) \leq$ $d_{K}^{n}(x, z) \max \left(d_{K}^{n}(x, y), d_{K}^{n}\left(x,\left(\tilde{K}^{n}\right)^{c}\right), d_{K}^{n}\left(y,\left(\tilde{K}^{n}\right)^{c}\right)\right)$. With the help of $(i i)$ the remaining cases may be lightly written.

The Borel $\sigma$-field of $B_{K}^{n}$ is denoted by $B f\left(B_{K}^{n}\right)$. If $\langle S, \mathrm{~L}, m\rangle$ is the measure space, then its completion relative to $m$ is denoted $A f(S, m)$, where $S$ is a set, $\mathrm{L}$ is a $\sigma$-algebra of subsets of $S, m$ is a real non-negative $\sigma$-finite measure on $\mathrm{L}$. That is, the $\sigma$-algebra $A f(S, m)$ contains all subsets $A \subset B$ of $B \in \mathrm{L}$ for which $m(B)=0$. In the non-Archimedean case the valuation group $\Gamma_{\mathbf{L}}^{\prime}:=\left\{|x|_{\mathbf{L}}: 0 \neq x \in \mathbf{L}\right\}$ of the local field $\mathbf{L}$ is discrete in $(0, \infty)$, hence subsets $U_{\epsilon}(y):=\{x \in K: d(x, y)<\epsilon\}$ are clopen (closed and open simultaneously) in $K=X$. Therefore, in the non-Archimedean case lemmas 1.1 and 1.2 from [36] have the following stronger forms.

2.2. Lemma. For an ultrametric space $(X, d)$ from $\S 2.1$ if $U$ is a clopen set in $X$, then $\left\{\gamma: N_{U}(\gamma) \geq l\right\}$ is also clopen in $X$ for each $l \in \mathbf{N}_{\mathbf{o}}$.

2.3. Lemma. For an ultrametric space $(X, d)$ from $\S 2.1$ and each $\epsilon>0$ and each $\gamma \in B_{X}^{n}$ there exists a clopen subset $O_{\epsilon}(\gamma)$ which belongs to the smallest $\sigma$-algebra B for which functions $N_{B}$ are measurable such that $\gamma \in$ $O_{\epsilon}(\gamma) \subset\left\{\gamma^{\prime}: d_{X}^{(n)}\left(\gamma, \gamma^{\prime}\right)<\epsilon\right\}$. 
Proof. For $\gamma=\left\{x_{1}, \ldots, x_{n}\right\}$ take $\eta \in \Gamma_{\mathbf{L}}^{\prime}$ such that $\epsilon>\eta>0$ and $U_{\eta p^{-n}}\left(x_{i}\right) \cap U_{\eta p^{-n}}\left(x_{j}\right)=\emptyset$ for each $i \neq j$. Put $O_{\epsilon}(\gamma):=\left\{\bigcap_{i=1}^{n}\left\{\gamma^{\prime}: \operatorname{card}\left(\gamma^{\prime} \cap\right.\right.\right.$ $\left.\left.U_{\eta p^{-n}}\left(x_{i}\right)\right) \geq 1\right\}$, where $1<p \in \Gamma_{\mathbf{L}}, p^{-1}=\left|\pi_{\mathbf{L}}\right|_{\mathbf{L}}, B\left(\mathbf{L}, 0,1^{-}\right)=\pi_{\mathbf{L}} B(\mathbf{L}, 0,1)$, $B(Y, x, r):=\left\{z \in Y: d_{Y}(x, z) \leq r\right\}, B\left(Y, y, r^{-}\right):=\left\{z \in Y: d_{Y}(y, z)<r\right\}$ for an ultrametric space $Y$ with an ultrametric $d_{Y}$.

2.4. Notes and definitions. Then theorems 1.1 and 1.2 from 36 are also true for all cases considered here. For this we mention, that as usually let $B_{K}:=\bigoplus_{n=0}^{\infty} B_{K}^{n}$, where $B_{K}^{0}:=\{\emptyset\}$ is a singleton. Since $X$ from $\S 2.1$ is not compact, then there exists an increasing sequence of subsets $K_{n} \subset X$ such that $X=\bigcup_{n} K_{n}$ and $K_{n}$ are Polish spaces in the induced topology from $X$. Moreover, $K_{n}$ can be chosen clopen in $X$ in the non-Archimedean case. Then the following space $\Gamma_{X}:=\left\{\gamma: \gamma \subset X\right.$ and $\operatorname{card}\left(\gamma \cap K_{n}\right)<\infty$ for each $\left.n\right\}$ is called the configuration space and it is isomorphic with the projective limit $p r-\lim \left\{B_{K_{n}}, \pi_{m}^{n}, \mathbf{N}\right\}$, where $\pi_{m}^{n}\left(\gamma_{m}\right)=\gamma_{n}$ for each $m>n$ and $\gamma_{n} \in B_{K_{n}}$. If $d_{n}$ denotes the metric in $B_{K_{n}}$, then $\left.d_{n+1}\right|_{B_{K_{n}}}=d_{n}$, since $K_{n} \subset K_{n+1}$. Then $\prod_{n=1}^{\infty} B_{K_{n}}=: Y$ in the Tychonoff product topology is metrizable, that induces the metric in $\Gamma_{X}$. Moreover, in the non-Archimedean case the metric $\rho$ in $Y$ can be chosen satisfying the ultrametric inequality: $\rho(x, y):=d_{n}\left(x_{n}, y_{n}\right) p^{-n}$, where $n=n(x, y):=\min _{\left(x_{j} \neq y_{j}\right)} j, x=\left(x_{j}: j \in \mathbf{N}, x_{j} \in B_{K_{j}}\right)$.

As it was proved in the papers cited in $\S 1$, on $X$ from $\S 2.1$ there exist real measures $m$ quasi-invariant relative to the left action of the corresponding group $G^{\prime}$ such that $m\left(K_{n}\right)<\infty$. In the case $X=G$, then $G^{\prime}$ is a dense subgroup in $G$. Quasi-invariance of $m$ implies, that $m$ are non-atomic. Let $K \in\left\{K_{n}: n \in \mathbf{N}\right\}$, then $m_{K}$ denotes the restriction $\left.m\right|_{K}$. Then $m_{K}^{n}:=\bigotimes_{j=1}^{n}\left(m_{K}\right)_{j}$ is a measure on $K^{n}$ and hence on $\tilde{K}^{n}$, since $m$ are non-atomic, where $\left(m_{K}\right)_{j}=m_{K}$ for each $j$. Therefore, $P_{K, m}:=$ $\exp (-m(K)) \sum_{n=0}^{\infty} m_{K, n} / n$ ! is a probability measure on $B f\left(B_{K}\right)$, where $m_{K, 0}$ is a probability measure on the singleton $B_{K}^{0}$, and $m_{K, n}$ are images of $m_{K}^{n}$ under the following mappings: $p_{K}^{n}:\left(x_{1}, \ldots, x_{n}\right) \in \tilde{K}^{n} \rightarrow\left\{x_{1}, \ldots, x_{n}\right\} \in B_{K}^{n}$. It was shown in $\S 1.2$ [36] that such system of measures $P_{K, n}$ is consistent, that is, $\pi_{l}^{n} P_{K_{l}, m}=P_{K_{n}, m}$ for each $n \leq l$. This defines the unique measure $P_{m}$ on $B f\left(\Gamma_{X}\right)$, which is called the Poisson measure. For each $n_{1}, . ., n_{l} \in \mathbf{N}_{\mathbf{o}}$ and disjoint Borel subsets $B_{1}, \ldots, B_{l}$ in $X$ there is the following equality:

(i) $P_{m}\left(\bigcap_{j=1}^{l}\left\{\gamma: \operatorname{card}\left(\gamma \cap B_{i}\right)=n_{i}\right\}\right)=\prod_{i=1}^{l} m\left(B_{i}\right)^{n_{i}} \exp \left(-m\left(B_{i}\right)\right) / n_{i}$ !

The configuration space $\Gamma_{X}$ consists of $\gamma \subset X$ such that $\operatorname{card}\left(\gamma \cap K_{n}\right)<\aleph_{0}$ for each $n \in \mathbf{N}$. In the case of $\operatorname{Diff}^{t}(M)$ this means that we need to consider such elements $g$ of this group for which $\operatorname{supp}(g) \subset K_{n}$ for some 
$n \in \mathbf{N}$, for example, a subgroup with supports of its elements contained in the corresponding finite unions of charts, where $\operatorname{supp}(g):=\operatorname{cl}\{x \in M$ : $g(x) \neq x\}$. Such subgroups are not Banach manifolds and they are denoted by $\operatorname{Diff}_{l}(M)$. In the case of $X=G$ the initial configuration space $\Gamma_{X}$ is not preserved by $G^{\prime}$, since there are $g \in G^{\prime}$ such that $g K_{n}$ is not contained in any $K_{m}$, because $\operatorname{supp}\left(L_{h}\right)=G$ for each $e \neq h \in G^{\prime}$, where $L_{h} g:=h g$ denotes the left shift in $G$ for $g, h \in G$.

Actually it is necessary to use more general construction in the case of $X=G$. Let $\tilde{\Gamma}_{X}:=\left[\bigcup_{g \in G^{\prime}} g \Gamma_{X}\right] / R$, where $R$ is an equivalence relation: $(g \gamma) R\left(g^{\prime} \gamma^{\prime}\right)$ if and only if $(g \gamma)=\left(g^{\prime} \gamma^{\prime}\right)$, where $\left[\bigcup_{g \in G^{\prime}} g \Gamma_{X}\right]$ is considered as the subset of $X^{\mathbf{N}}$. The group $G^{\prime}$ is separable, hence there exists a countable dense subset $\left\{g_{j}: j \in \mathbf{N}\right\}$. To each element $g \in G^{\prime}$ there corresponds a subsequence $\left\{g_{j_{n}}: n \in \mathbf{N}\right\}$ converging to $g$ in $G^{\prime}$. Hence each $g \gamma$ is completely characterised by the corresponding subsequence $\left\{g_{j_{n}} \gamma: n \in \mathbf{N}\right\}$. Therefore, $\tilde{\Gamma}_{X}$ has the embedding into $X^{\mathbf{N}}$ as the closed subset, since the family

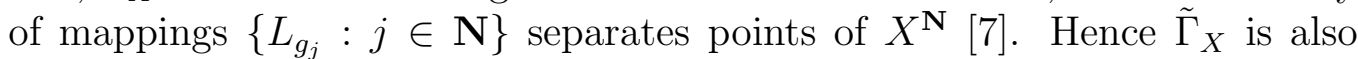
metrizable and complete. The manifold $\tilde{\Gamma}_{X}$ for each its point has a neighbourhood diffeomorphic with the corresponding open subset of $\Gamma_{X}$, since for each $K_{n}$ there exist a neighbourhood $U_{n}^{\prime}$ of $e$ in $G^{\prime}$ and $m>n$ such that $U_{n}^{\prime} K_{n} \subset K_{m}$. A choice of such sequence $K_{n} \subset \operatorname{Int}\left(K_{n+1}\right)$ with canonical closed subsets $K_{n}$ is given independently in $\S 2.9$. The manifold $\tilde{\Gamma}_{X}$ is paracompact, consequently, it has a locally finite covering $\left\{S_{l}: l \in \mathbf{N}\right\}$, where $S_{l}$ are open in $\tilde{\Gamma}_{X}$ and diffeomorphic with the corresponding open subsets $Q_{l}$ of $\Gamma_{X}$ for which $P_{m}\left(Q_{l}\right)<\infty, \zeta_{l}: S_{l} \rightarrow Q_{l}$ denote such diffeomorphisms. This means that the Poisson measure $P_{m}$ on $\Gamma_{X}$ induces the corresponding $\sigma$-additive $\sigma$-finite quasi-invariant relative to $G^{\prime}$ measure $\mu$ on $\tilde{\Gamma}_{X}$ such that $\mu(E):=C \sum_{l} P_{m}\left(\zeta_{l}\left(E \cap S_{l}\right)\right) 2^{-l}$ which is also denoted by $P_{m}$, where $E \in B f\left(\tilde{\Gamma}_{X}\right)$, a constant $C>0$ is chosen such that $\mu\left(\tilde{\Gamma}_{X}\right)=1$. Therefore, $P_{m}$ on $\tilde{\Gamma}_{X}$ is the probability measure as also for the case $\Gamma_{M}$ for $\operatorname{dim}_{\mathbf{L}} M<\infty$. This gives possibility to consider the case $X=G$ as well as the case $X=M$ for groups of diffeomorphisms $\operatorname{Diff}^{t}(M)$ of class $C^{t}$ with $1 \leq t \leq \infty$, which have structure of Banach manifolds from the papers cited above.

If the manifold $M$ is locally compact and each $K_{n}$ is chosen to be canonical closed compact subset, then for $\operatorname{Diff} f^{t}(M)$ the configuration spaces $\Gamma_{M}$ and $\tilde{\Gamma}_{M}$ coincide. Indeed, if $\gamma \in \Gamma_{M}$, then $\operatorname{card}\left(\gamma \cap K_{n}\right)<\aleph_{0}$ for each $n \in \mathbf{N}$. Each subset $K_{n}$ is compact and canonically closed, hence is sequen- 
tially compact [7]. This means that if $\operatorname{card}\left((g \gamma) \cap K_{l}\right)=\aleph_{0}$ for some $l \in \mathbf{N}$ and $g \in \operatorname{Diff}^{t}(M)$, then $\left\{g \gamma_{j}: j \in \mathbf{N}\right\}$ contains a convergent subsequence in $K_{l}$. But $\left\{\gamma_{j}: j \in \mathbf{N}\right\}=\gamma$ is the disrete subset of $M$, hence $g^{-1}$ is not continuous, since $\left\{g \gamma_{j}: j \in \mathbf{N}\right\}$ is not closed in $M$. This contradicts supposition $g \in \operatorname{Diff}^{t}(M)$, consequently, $g \gamma \in \Gamma_{M}$ for each $g \in \operatorname{Diff}^{t}(M)$ for locally compact $M$ and canonical closed compact subsets $K_{n}$ in $M$. Therefore, $\bigcup_{g \in \operatorname{Diff}^{t}(M)} g \Gamma_{M}=\Gamma_{M}$, since $g \Gamma_{M} \subset \Gamma_{M}$ for each $g \in \operatorname{Diff}^{t}(M)$ and $e \Gamma_{M}=\Gamma_{M}$, consequently, $\tilde{\Gamma}_{M}=\Gamma_{M}$.

If $M$ is not locally compact, for example, $M \backslash M_{R}=\bigcup_{j=1}^{\infty} \Omega_{j}$, where $\Omega_{j}$ are disjoint open subsets of $M, M_{R}:=\left\{x \in M: d_{M}\left(x, x_{0}\right) \leq R\right\}$, $0<R<\infty, x_{0}$ is a fixed point in $M$ and $d_{M}$ is a metric in $M$, then there exists $g \in \operatorname{Dif} f^{\infty}(M)$ with $\operatorname{supp}(g)$ bounded in $M$ and a bounded infinite sequence of $\gamma_{j} \in M \backslash M_{R}$ which is discrete in $M$, that is, $\operatorname{cl}\left\{\gamma_{j}: j \in \mathbf{N}\right\}=$ $\left\{\gamma_{j}: j \in \mathbf{N}\right\}$, such that $\operatorname{card}\left((g \gamma) \cap K_{n}\right)=\aleph_{0}$ for some canonically closed $K_{n}$ in $M$, since each $K_{n}$ is not locally compact, when $\operatorname{dim}_{\mathbf{L}} M=\infty$, where $\mathbf{L}$ is the corresponding field either $\mathbf{R}$ or the local field. Hence in this case $\tilde{\Gamma}_{M} \neq \Gamma_{M}$.

If $X=G$, then in view of the choice of $K_{n}$ in $\S 2.9$ that to fulfil demands on the measure $m$, there exists $g \in G^{\prime}$ and $n \in \mathbf{N}$ such that $g K_{n}$ is not contained in each $K_{l}$, where $l \in \mathbf{N}$. This $g$ can be chosen by induction, since $K_{l}$ are not locally compact for each $l$ and $G$ is not locally compact. Therefore, there exists a discrete infinite sequence $\gamma$ in $g K_{n}$ such that $\operatorname{card}\left(\gamma \cap K_{l}\right)<\aleph_{0}$ for each $l \in \mathbf{N}$. But $\gamma \in \Gamma_{G}$ and $g^{-1} \in G^{\prime}$ and $g^{-1} \gamma \in \tilde{\Gamma}_{G} \backslash \Gamma_{G}$, since $\operatorname{card}\left(\left(g^{-1} \gamma\right) \cap K_{n}\right)=\aleph_{0}$. Hence $\tilde{\Gamma}_{G} \neq \Gamma_{G}$ in this case also.

The group $G^{\prime}$ and $X$ and $\Gamma_{X}$ have structures of the $C^{\infty}$-manifolds, since $X$ is the $C^{\infty}$-manifold. Therefore, $\tilde{\Gamma}_{X}$ is the $C^{\infty}$-manifold also. In the nonArchimedean case $M, G^{\prime}, G$ and hence $\Gamma_{X}$ and $\tilde{\Gamma}_{X}$ are analytic manifolds with disjoint clopen charts, since $\Gamma^{\prime}{ }_{\mathbf{L}}$ is discrete in $(0, \infty)$ and $\Gamma_{X}$ and $\tilde{\Gamma}_{X}$ are infinite-dimensional over $\mathbf{L}$ [22].

It is necessary to note, that for $X=G$ the dense subgroup $G^{\prime}$ acts by the left shifts $L_{h}: G \rightarrow G$ as the diffeomorphism for each $h \in G^{\prime}$, where $G$ is either the loop group or the diffeomorphisms group. Therefore, lemmas 2.1, 2.2 and theorems 2.1, 2.2 and 2.3 from [36] are applicable to the cases considered here, since $\Gamma_{X}$ produces charts for $\Gamma_{X}$ and $P_{m}$ on $\Gamma_{X}$ induces $P_{m}$ on $\tilde{\Gamma}_{X}$. Theorem 2.3 from 36 can be applied to the real and non-Archimedean cases of $X=M$. 
2.5. Definition. (see [9] \$19.3.) Let $G^{\prime}$ be a group acting from the left on the measure space $\langle X, \mathrm{~L}, m\rangle$. Then $\langle X, \mathrm{~L}, m\rangle$ is called a measure $G^{\prime}$-transformation space if $(i) x W \in \mathrm{L}$ whenever $x \in G^{\prime}$ and $W \in \mathrm{L}$, and (ii) $m(x W)=0$ whenever $x \in G^{\prime}, W \in \mathrm{L}$ and $m(W)=0$.

2.6. Note. For the considered here cases and $B f(X) \subset \mathrm{L}$ conditions of definition 2.5 are fulfilled for the quasi-invariant measure $m$ on $X$ relative to $G^{\prime}$.

2.7. Definition. The measure $G^{\prime}$-transformation space $\langle S, \mathrm{~L}, m\rangle$ is ergodic under $G^{\prime}$ if, whenever, $V, W \in \mathrm{L}$ with $m(V) m(W) \neq 0$, there exists $x \in G^{\prime}$ such that $m(x V \cap W) \neq 0$.

2.8. Note. It was proved in [18, 20, 21, 23, 24, 25] that $m$ on $X$ is ergodic under $G^{\prime}$ for the considered here cases $(1-3)$, since $m$ is quasiinvariant relative to $G^{\prime}$. In cases $(1,2)$ at first $m$ was constructed on a neighbourhood $W$ of $e$ in $G$. But theorem 2.3 from [36] can not be applied to the cases $X=G$ for the probability measure $m$ on $X$, since in view of the construction of the Gaussian measure $m$ on $G$ there are $\epsilon>0$ and $n \in \mathbf{N}$ such that for each $\psi \in G^{\prime}$ with $\psi\left(K_{n}\right) \cap K_{n}=\emptyset$ the following integral is rather large: $\int_{G}\left|\rho_{m}^{1 / 2}(\psi, x)-1\right| m(d x)>\epsilon$, where $m^{\psi}(E):=m\left(\psi^{-1} E\right)$ for each $E \in A f(X, m), \rho_{m}(\psi, x):=m^{\psi}(d x) / m(d x)$.

There are locally finite coverings $\left\{g_{j} W_{j}: j \in \mathbf{N}_{\mathbf{o}}\right\}$ of $G$ and $\left\{g_{j} W_{j}^{\prime}\right.$ : $\left.j \in \mathbf{N}_{\mathbf{o}}\right\}$ of $G^{\prime}$, since $G$ and $G^{\prime}$ are paracompact spaces relative to their own topologies $\tau$ and $\tau^{\prime}$ respectively and $G^{\prime}$ is dense in $G$, where $W_{0}=W$, $W_{j} \subset W$ for each $j, W^{\prime} \subset W \cap G^{\prime}, W_{0}^{\prime}=W^{\prime}, W_{j}^{\prime} \subset W^{\prime}$ for each $j, g_{j} \in G^{\prime}$ for each $j, g_{0}=e, W_{j}$ are open in $G$ and $W_{j}^{\prime}$ are open in $G^{\prime}$. Analogously for the pair $G^{\prime}$ and $X=M$ in cases $(3)(a, b)$. Then $m$ on $W$ can be extended as a $\sigma$-finite measure on $B f(G)$ by the formula:

(i) $m(V):=\sum_{j=0}^{\infty} m\left(g_{j}^{-1}\left(V \cap g_{j} W_{j}\right)\right)$, since $0<m(W)<\infty$. The group $G$ is not locally compact, hence $m(G)=\infty$. Using analogous procedure with a locally finite covering $\left\{g_{j} W_{j}: j \in \mathbf{N}_{\mathbf{o}}\right\}$ with $W_{j}$ open in $M$ and a neighbourhood $W$ of a marked point $x_{0} \in M$ without relation between $W^{\prime}$ and $W$ we get a $\sigma$-finite measure $m$ on $M$ for non-locally compact manifold $M$ with $m(M)=\infty$. We choose in these cases $m\left(K_{n}\right)<\infty$ for each $n \in \mathbf{N}$. As follows from the cited papers it is possible to choose $K_{n} \subset \cup_{j=0}^{n} g_{j} W_{j}$ and $m$ such that

(ii) for each $\epsilon>0$ and each $n \in \mathbf{N}$ there exists $\psi \in G^{\prime}$ such that $\psi\left(K_{n}\right) \cap$ $K_{n}=\emptyset$ and $\bigcup_{n} K_{n}=X$ and $\int_{X}\left|\rho_{m}^{1 / 2}(\psi, x)-1\right|^{2} m(d x)<\epsilon$. Then it is proved below in theorem 2.9 that such $m$ exists and $P_{m}$ on $\tilde{\Gamma}_{X}$ is ergodic. 
Henceforth, such $\sigma$-finite measures $m$ on $X$ are used with $m(X)=\infty$, since for $m(X)=1$ the corresponding measures $P_{m}$ are not ergodic (see note after definiton 1 in $\S 2$ [36]).

2.9. Theorem. There exist quasi-invariant $\sigma$-finite measures $m$ on $X$ relative to the groups $G^{\prime}$ with $m(X)=\infty$ satisfying condition (ii) from §2.8. For such $m$ the Poisson measure $P_{m}$ on $\tilde{\Gamma}_{X}$ is ergodic .

Proof. To prove $P_{m}$ is ergodic on $\Gamma_{X}$ we use the fact, that $m$ is ergodic on $X$. The measure space $\langle S, \mathrm{~L}, m\rangle$ is said to have property $(P)$ if, for any locally $m$-measurable subset $W$ of $S$ such that $x W \ominus W$ is locally $m$-null for each $x \in G^{\prime}$, either $W$ is locally $m$-null or $S \backslash W$ is locally $m$ null. The measure space $\langle S, \mathrm{~L}, m\rangle$ is called parabounded if there exists a pairwise disjoint subfamily $\mathrm{W}$ of $\mathrm{L}$ such that $(i)$ for each $A \in \mathrm{L},\{B \in \mathrm{W}$ : $A \cap B \neq \emptyset\}$ is countable, and (ii) $X \backslash \bigcup_{W \in \mathrm{W}} W$ is locally $m$-null. It was proved in proposition 19.5 [9] that if $\langle S, \mathrm{~L}, m\rangle$ is ergodic it has property $(P)$. Conversely, if $\langle S, \mathrm{~L}, m>$ has property $(P)$ and is parabounded, it is ergodic. The space $\Gamma_{X}$ is isomorphic with the projective limit $p r-\lim \left\{B_{K_{n}}, \pi_{m}^{n}, \mathbf{N}\right\}$, which is the closed subset in $\prod_{n} B_{K_{n}}$. The latter is the Polish space, hence $\tilde{\Gamma}_{X}$ is the Polish space [7]. The measure spaces $\langle X, A f(X, m), m\rangle$ and $<\tilde{\Gamma}_{X}, A f\left(\tilde{\Gamma}_{X}, P_{m}\right), P_{m}>$ are parabounded, since $X$ and $\tilde{\Gamma}_{X}$ are the Polish spaces and hence are the Radon spaces (see chapter 1 in [5]), that is, the class of compact subsets approximates from below the corresponding measures $\left.m\right|_{K_{n}}$ and $P_{m}$. Therefore, it remains to show, that $<\tilde{\Gamma}_{X}, A f\left(\tilde{\Gamma}_{X}, P_{m}\right), P_{m}>$ has property $(P)$. But this follows from theorem 2.3 [36] and $\$ 2.8$, if to show that condition 2.8 (ii) is fulfilled for $m$ and $U_{n}^{\prime} K_{n} \subset \operatorname{Int}\left(K_{n+1}\right)$ for the corresponding $K_{n}$ in $X$ and neighbourhoods $U_{n}^{\prime}$ of $e$ in $G^{\prime}$, since there are the local diffeomorphisms $\zeta_{l}: S_{l} \rightarrow Q_{l}$ from $\S 2.4$ and $P_{m}$ and $m$ are $\sigma$-finite measures. In this situation integral equalities and inequalities from the proof of theorem 2.3 [36] are transferable onto the case of $\tilde{\Gamma}_{X}$ considered here.

For the construction of such $m$ take it at first on an open subset $U \subset X$ such that $W$ is sufficiently small: $W^{\prime} W \subset U$. In the case of $G=X$ in addition let $e \in U$ and $U^{-1}=U, W^{-1}=W, W^{-1}=W^{\prime}$ (see references in §2.8). The quasi-invariance factor $\rho_{m}(x, y)$ is continuos on $W^{\prime} \times W$ and $\rho_{m}(e, y)=1$, where $\rho_{m}(x, y):=m^{x}(d y) / m(d y), m^{x}(A):=m\left(x^{-1} A\right)$ for each $x \in G^{\prime}$ and $A \in A f(W, m)$. Take open subsets $W_{0} \subset W$ and $W_{0}^{\prime} \subset W^{\prime}$ for which $\left|\rho_{m}(x, y)-1\right|<1$ for each $(x, y) \in W_{0}^{\prime} \times W_{0}$.

The measure $m$ is regular and approximated from above by the class of open subsets [5, 9]. Therefore, it is possible to choose by induction open 
subsets $W_{j} \subset W_{0}$ and $e \in W_{j}^{\prime} \subset W_{0}^{\prime}$ and a sequence of elements $g_{j} \in G^{\prime}$ such that $m\left(g_{j}^{-1}\left(g_{j} W_{j} \cap\left[\bigcup_{i=1}^{j-1} g_{i} W_{i}\right]\right)\right)<2^{-j}$ and $\left|\rho_{w}(x, y)-1\right|<2^{-j}$ on $g_{j} W_{j}^{\prime} \times g_{j} W_{j}$, where $w$ is a measure on $B f\left(g_{j} W_{j}\right)$ defined by the following formula $w\left(g_{j} A\right):=m(A)$ for each $A \in B f\left(W_{j}\right), g_{0}=e$. Then $m$ on $B f(X)$ is defined by formula 2.8(i) and certainly has the extension $m$ onto $A f(X, m)$.

The measure $m$ is induced from the corresponding measure $\lambda$ on the Banach space $Y$ due to the local diffeomorphism $A: U \rightarrow V$, where $V$ is an open neighbourhood of 0 in $Y$ and $U$ is open in $X$. From the quasi-invariance of $\lambda$ relative to shifts from a dense subspace $Y^{\prime}$ it follows a property:

$(\alpha)$ for each Borel subset $E \subset Y$ which is a $C^{1}$-submanifold in $Y$ of codimension 1 in $Y$ (over the field $\mathbf{R}$ or the non-Archimedean local field) such that $T_{y} E$ is not subset of $Y^{\prime}$ for each $y \in E$ it follows that $\lambda(E)=0$, since $\lambda$ is the quasi-invariant non-negative $\sigma$-additive and $\sigma$-finite measure. In particular, for finite-dimensional $X=M$ over the corresponding field the space $Y$ is finite-dimensional and $\lambda$ can be taken as the Haar measure on $Y$ (in the real case it concides with the Lebesgue measure). For infinitedimensional real $X$, particularly for $X=G$, the measure $\lambda$ can be taken Gaussian. For infinite-dimensional $X$ over the local field the wider class of measures $\lambda$ was constructed in the papers cited in $\S 1$. Then we choose (take) by induction a sequence $K_{n} \subset \bigcup_{i=0}^{n} g_{i} W_{i}$ satisfying the following conditions $U^{\prime}{ }_{n} K_{n} \subset \operatorname{Int}\left(K_{n+1}\right)$ for each $n$ with $\bigcup_{n} K_{n}=X$ and $m\left(K_{n} \backslash \operatorname{Int}\left(K_{n}\right)\right)=0$ and $K_{n}$ are canonical closed subsets, that is, $\operatorname{cl}\left(\operatorname{Int}\left(K_{n}\right)\right)=K_{n}$, since $m$ is quasi-invariant and has not any atoms and due to property $(\alpha)$ of $\lambda$, where $\operatorname{cl}(A)$ denotes the closure of a subset $A \subset X$ in $X, \operatorname{Int}(A)$ denotes the interior of $A$ in $X, U_{n}^{\prime}$ are the corresponding (open) neighbourhoods of $e$ in $G^{\prime}$ such that $U_{n}^{\prime} \subset W^{\prime}$. The space $X$ is Polish, hence each $K_{n}$ is the Polish topological subspace [0]. Certainly, in the non-Archimedean cases each $K_{n}$ can be chosen clopen (closed and open) in $X$, that is, $\operatorname{Int}\left(K_{n}\right)=K_{n}=\operatorname{cl}\left(K_{n}\right)$, since the base of the topology of $X$ consists of clopen subsets. Since $X$ is not locally compact, then there exists the sequence $\left\{K_{n}: n \in \mathbf{N}\right\}$ fulfilling condition 2.8(ii).

2.10. Note. In cases $(3)(a, b)$ for $X=M$ and $G^{\prime}=\operatorname{Diff}^{t}(M)$ in addition we have the following.

2.11. Definition. Let $G_{K_{n}}^{\prime}:=\left\{\psi \in G^{\prime}:\left.\psi\right|_{K_{n}^{c}}=i d\right\}$ and let $f$ be a symmetric measurable function defined on $\tilde{K}_{n}^{l}$, where $l \in \mathbf{N}, A^{c}:=X \backslash A$ for a subset $A$ in $X, K_{n}$ are canonical closed subsets with $\bigcup_{n} K_{n}=X$ and $K_{n} \subset$ 
$K_{n+1}$ and $m\left(K_{n} \backslash \operatorname{Int}\left(K_{n}\right)\right)=0$ for each $n \in \mathbf{N}$. In the non-Archimedean case let also $K_{n}$ be clopen in $M$, which automatically implies $K_{n} \backslash \operatorname{Int}\left(K_{n}\right)=\emptyset$. The measure $m$ is called $G^{\prime l} K_{n}$-ergodic, if $f$ is constant modulo null sets, then $f\left(x_{1}, \ldots, x_{l}\right)=f\left(\psi\left(x_{1}\right), \ldots, \psi\left(x_{l}\right)\right)$ for $m_{K_{n}}^{l}$-a.e $x=\left(x_{1}, \ldots, x_{l}\right)$ for all $\psi \in G^{\prime}$.

2.12. Theorem. If for each $n$ the measure $m$ is $G_{K_{n}}^{\prime l}$-ergodic for some $N \geq n$ and all $l$, then $P_{m}$ is $G^{\prime}$-ergodic.

Proof. As it was shown in papers [18, 21, 22, 29, 30] the subgroups $G^{\prime} K_{n}$ are correctly defined for canonical closed subsets $K_{n}$ in $M=X, G^{\prime} K_{n} \subset G^{\prime}$ for each $n$, since from $\left.\psi\right|_{K_{n}^{c}}=i d$ it follows, that $\left.\psi\right|_{c l\left(K_{n}^{c}\right)}=i d$. The rest of the proof is as in the proof of theorem 2.4 [36], which can be applied locally and then with the help of the local diffeomorphisms $\zeta_{l}: S_{l} \rightarrow Q_{l}$ is extendable onto the case of $\tilde{\Gamma}_{X}$ considered here, since $G^{\prime l}{ }_{K_{N}} \tilde{K}_{N}^{l}=\tilde{K}_{N}^{l}$ and for the measure $\nu(A):=P_{m}(E \cap A)$ for each $A \in B f\left(\tilde{\Gamma}_{X}\right)$ we have $\nu(B)=\int_{0}^{\infty} P_{c m}(B) \lambda(d c)$ for each $B \in B f\left(\Gamma_{X}\right)$, where $c \geq 0$ and $\lambda$ is a suitable Borel measure on $[0, \infty)$. From $P_{m}\left(\zeta_{l}\left(A \cap S_{l}\right)\right)=0$ for each $l$ it follows, that $P_{m}(A)=0$. Thus if $\lambda(\{1\})>0$, then $P_{m}(A)=0$; if $\lambda(\{1\})=0$, then $P_{m}\left(A^{c}\right)=0$, where $A$ is a $P_{m}$-measurable subset of $\tilde{\Gamma}_{X}$ for which $P_{m}\left(A \triangle \psi^{-1} A\right)=0$ for all $\psi \in G^{\prime}$, where $A \triangle B:=(A \backslash B) \cup(B \backslash A))$.

2.13. Note. From theorem 2.12 it can be deduced in another way, than it was done in theorem 2.9 , that $P_{m}$ on $\tilde{\Gamma}_{X}$ is $G^{\prime}$-ergodic in cases $(3)(a, b)$ for $X=M$, when $m$ and $K_{n}$ are chosen in accordance with $\S 2.8$ and $\S 2.11$. The proof of this is analogous to that of theorem 2.5 [36], since $m$ is ergodic and quasi-invariant with the continuous quasi-invariance factor $\rho_{m}(\psi, x)$ on $G^{\prime} \times X, m(X)=\infty$ and $m\left(K_{n} \backslash \operatorname{Int}\left(K_{n}\right)\right)=0$, since due to $\S 2.4$ there are the local diffeomorphisms $\zeta_{l}: S_{l} \rightarrow Q_{l}$ and $\operatorname{Diff}^{t}(X ; K) \tilde{K}^{l}=\tilde{K}^{l}$ for each canonical closed subset $K$ in $X$, where $\operatorname{Diff}^{t}(X ; K):=\left\{f \in \operatorname{Diff}^{t}(X)\right.$ : $\left.\left.f\right|_{K^{c}}=i d\right\}$.

2.14. Lemma. Let $Y$ be a canonically closed subset in $X, Y \subset K_{n}$ for some $n \in \mathbf{N}$. Suppose that $\mu$ is a quasi-invariant measure on $\tilde{\Gamma}_{X}$ relative to $G^{\prime}=\operatorname{Diff}^{t}(X)$ for a $C^{\infty}$-manifold $X=M$ (in the non-Archimedean case an analytic manifold $M$ ) and $\mu_{n}$ be a restriction of $\mu$ on $B_{Y}^{n} \times \tilde{\Gamma}_{X \backslash Y}$ and $\mu_{n}^{\prime}$ and $\mu_{n}{ }_{n}$ be projections of $\mu_{n}$ on $B_{Y}^{n}$ and $\tilde{\Gamma}_{X \backslash Y}$ respectively. Then $\mu_{n}$ is equivalent with $\mu_{n}^{\prime} \times \mu^{\prime \prime}{ }_{n}$. In the non-Archimedean case this is also true for $Y$ clopen in $X$.

Proof. In view of $\S 2.9 m(Y \backslash \operatorname{Int}(Y))=0$. The group $\operatorname{Diff}^{t}(X ; X \backslash Y)$ is a closed subgroup of $\operatorname{Diff}^{t}(X)$, hence $\tilde{\Gamma}_{X \backslash Y}=\left(\operatorname{Diff}^{t}(X ; X \backslash Y) \Gamma_{X}\right) / R$ 
is a $C^{\infty}$-submanifold of $\tilde{\Gamma}_{X}$ (see also $\left.\S 2.4\right)$. The measures $\mu_{n}^{\prime} \times \mu^{\prime \prime}{ }_{n}$ with $\mu_{n}$ are equivalent if and only if $\mu$ and $\mu^{\prime} \times \mu$ " are equivalent, since $\mu$ is quasiinvariant relative to $G^{\prime}$ and non-atomic, where $\mu^{\prime}$ is a projection of $\left.\mu\right|_{B_{Y} \times \tilde{\Gamma}_{X \backslash Y}}$ on $B_{Y}$ and $\mu$ " is a projection of $\mu$ on $\tilde{\Gamma}_{X \backslash Y}$. On the other hand, $G^{\prime} B_{X}^{n}=B_{X}^{n}$ for each $n \in \mathbf{N}$ and $G^{\prime} \tilde{\Gamma}_{X}=\tilde{\Gamma}_{X}$, also Diff $(X ; X \backslash Y) \tilde{\Gamma}_{X \backslash Y}=\tilde{\Gamma}_{X \backslash Y}$. On the other hand, $B_{Y}^{n} \times \tilde{\Gamma}_{X \backslash Y}$ is the Borel subset of $\tilde{\Gamma}_{X}$, since $B_{Y}^{n}$ is the Borel subset of $\Gamma_{X}$. For the rest of the proof are necessary two propositions.

2.15. Proposition. In the group Diff $f^{t}(\operatorname{Int}(Y))$ there exists a countable family of one-parameter subgroups $G_{l}$ such that generated by them group $J \subset$ Dif $f^{t}(Y)$ acts transitively on $B_{Y}^{n}$.

Proof. For Diff $f^{t}(\operatorname{Int}(Y))$ one-parameter subgroups can be chosen as in proposition 2.1 [38] with the help of [6] and theorems about existence of oneparameter subgroups of $\operatorname{Diff}^{t}(Y)$ for infinite-dimensional Banach manifolds $M$ from [17, 21, 26, 27], where one-parameter subgroups are real for real $M$ and $g^{b}$ with $b \in \mathbf{L}$ for $M$ over the local field $\mathbf{L}$ such that $g^{a} g^{b}=g^{a+b}$ for each $a, b \in \mathbf{L}$. In the non-Archimedean case one-parameter subgroups can aslo be indexed by $b \in B(\mathbf{L}, 0,1)$, where $B(S, x, r):=\left\{y \in S: d_{S}(x, y) \leq r\right\}$ denotes a ball in a metric space $S$ with a metric $d_{S}$ and a point $x \in S$. This is possible, since $M$ and $T_{x} M$ are separable spaces for each $x \in M$ and using countable atlas $\operatorname{At}(M)=\left\{\left(U_{j}, \phi_{j}\right): j\right\}$ of $M$ and considering one-parameter subgroups with $\operatorname{supp}\left(g^{b}\right) \subset U_{j}$ for each $b \in \mathbf{L}$ for the corresponding chart $U_{j}$, where either $\mathbf{L}=\mathbf{R}$ or $\mathbf{L}$ is the local field, $U_{j}$ are open in $M$ and $\phi_{j}: U_{j} \rightarrow V_{j}$ are diffeomorphisms, $V_{j}$ are open in the corresponding Banach space.

2.16. Proposition. Let $\mathbf{L}$ or may be $B(\mathbf{L}, 0,1)$ also in the non-Archimedean case acts measurably in a measure space $(M, B f(M), \mu)$ such that $\mu$ is quasiinvarint relative to the action of $\mathbf{L}$ or $B(\mathbf{L}, 0,1)$ on $M$, where $M$ is a $C^{\infty}$ manifold (analytic in the non-Archimedean case) and $\mu$ is induced by a quasiinvariant non-negative $\sigma$-additive and $\sigma$-finite measure $\eta$ relative to shifts from a dense subspace $Z^{\prime}$ and $\eta$ is on the Borel field $B f(Z)$ of the separable Banach space $Z$ over a field $\mathbf{L}$ which is either $\mathbf{L}=\mathbf{R}$ or a local field such that $Z=T_{x} M$ for each $x \in M$. Suppose that a partition $\zeta$ of $M$ is invariant by $\bmod (\mu)$ relative to the action of $\mathbf{L}$ or $B(\mathbf{L}, 0,1)$ on $M$ and projections of $\eta$ onto one-dimensional over $\mathbf{L}$ subspaces are equivalent with the non-negative Haar measure $\lambda$ on $\mathbf{L}$. Then for $\mu$-almost each $C \in \zeta$ the conditional measures $\mu^{C}$ are quasi-invariant relative to the action of $\mathbf{L}$ or $B(\mathbf{L}, 0,1)$ respectively. 
Proof. The proof is almost the same as in proposition 2.2 [38] with the substitution of $\mathbf{R}$ onto $\mathbf{L}$ or may be $B(\mathbf{L}, 0,1)$ in the non-Archimedean case and using the Haar measure $\lambda$ on a locally compact subgroup $S$ of $\mathbf{L}$ with $\lambda(\mathbf{L} \backslash S)=0$ or $\lambda(B(\mathbf{L}, 0,1) \backslash S)=0$, which implies $S=\mathbf{L}$ or $S=B(\mathbf{L}, 0,1)$ respectively by the A. Weil theorem, since each quasi-invariant measure on a locally compact group (relative to its action on itself) is equivalent with the Haar measure 4 .

Continuation of the proof of lemma 2.14. In view of proposition 2.15 there exists a subgroup $J$ which acts transitively on $B_{\operatorname{Int}(Y)}^{n}$. In view of proposition 2.16 from an isomorphism of one-parameter subgroup $G_{l}$ with $\mathbf{L}$ or $B(\mathbf{L}, 0,1)$ for $\mu^{\prime \prime}{ }_{n}$-a.e. configurations $\gamma \in \tilde{\Gamma}_{X \backslash Y}$ the conditional measure $\mu_{n}^{\gamma}$ on $B_{Y}^{n}$ is quasi-invariant relative to each one-parameter subgroup $G_{l}$, hence realtive to the minimal subgroup $J$ of $\operatorname{Diff}^{t}(X)$ generated by $\bigcup_{l=1}^{\infty} G_{l}$. The measure $\mu_{n}^{\gamma}$ on $B_{Y}^{n}$ induces the measure $\eta$ on $T_{\gamma^{n}} B_{Y}^{n}$ for each $\gamma^{n} \in$ $B_{Y}^{n}$. This measure $\eta$ is completely characterised by its finite-dimensional projections $\eta_{n}$ onto subspaces $F_{n}$ such that $F_{n} \subset F_{n+1}$ for each $n \in \mathbf{N}$ and $\bigcup_{n} F_{n}$ is dense in $T_{\gamma^{n}} B_{Y}^{n}$ (see about weak distributions [28, 37]). It is supposed that the manifold $M$ has the foliated structure such that $M_{n} \subset M_{n+1}$ and $\operatorname{dim}_{\mathbf{L}} M_{n}=k_{n}<\infty$ for each $n \in \mathbf{N}$ and $\bigcup_{n \in \mathbf{N}} M_{n}$ is dense in $M$. Theorefore, to $\mu_{n}^{\gamma}$ there corresponds a family of measures $\tilde{\eta}_{n}$ on $M_{n}$ with the help of a locally finite coverings and the exponential mapping exp $: \tilde{M} \rightarrow M$ from the neighbourhood $\tilde{T} M$ of $M$ in $T M$ onto $M$ such that $\exp _{x}: V_{x} \rightarrow W_{x}$ are local diffeomorphisms of open subsets $V_{x}$ in $T_{x} M$ and $W_{x}$ in $M$ with $x \in M$. A measure $\tilde{\eta}_{n}$ is quasi-invariant relative to $J_{n}:=\left\{g \in J: g_{M \backslash M_{n}}=i d\right\}$. The manifold $M_{n}$ is locally compact, hence $\tilde{\eta}_{n}$ is equivalent with the Riemann volume element on $M_{n}$ in the real case and with the restriction of the Haar measure from $\mathbf{L}^{k_{n}}$ onto $M_{n}$ in the non-Archimedean case, since in the latter case $M_{n}$ is embeddable into $\mathbf{L}^{k_{n}}$ due to a partition of $M_{n}$ into a disjoint union of balls. In view of the Kakutani theorem II.4.1 [5] $\mu_{n}$ is equivalent with $\mu_{n}^{\prime} \times \mu_{n}{ }_{n}$, since a finite measure $\zeta$ on $B f(A \times B)$ is equivalent with the direct product $\zeta_{A} \times \zeta_{B}$, where $\zeta_{A}$ and $\zeta_{B}$ are projections of $\zeta$ on Hausdorff topological spaces $A$ and $B$ respectively. 


\section{Unitary representations associated with the Poisson measures.}

3.1. Definitions and notes. Let $H:=L^{2}\left(\tilde{\Gamma}_{X}, P_{m}, \mathbf{C}\right)$ be the standard Hilbert space of equivalence classes of measurable functions $f: \tilde{\Gamma}_{X} \rightarrow \mathbf{C}$ for which $\|f\|_{H}^{2}:=\int_{\tilde{\Gamma}_{X}}|f(x)|^{2} P_{m}(d x)<\infty$, where $P_{m}$ is the Poisson measure given in $\S 2.4$. Then consider the following representation:

(i) $U_{m}(\psi) f(\gamma):=\rho_{P_{m}}^{1 / 2}(\psi, \gamma) f\left(\psi^{-1}(\gamma)\right)$, where $\rho_{P_{m}}(\psi, \gamma):=P_{m^{\psi}}(d \gamma) / P_{m}(d \gamma), \gamma \in \tilde{\Gamma}_{X}, f \in H, \psi \in G^{\prime}, m^{\psi}(E):=$ $m\left(\psi^{-1} E\right)$ for each $E \in A f(X, m)$. That is, $U_{m}: G^{\prime} \rightarrow U(H)$, where $U(H)$ is the unitary group of the Hilbert space $H$. The topology of $U(H)$ is induced by the operator norm in the space $L(H)$ of bounded linear operators $S: H \rightarrow H$, $d(A, B):=d\left(B^{-1} A, I\right):=\left\|B^{-1} A-I\right\|_{L(H)}$ is the metric in $U(H)$, where $A, B \in U(H), I$ denotes the unit operator on $H$.

In cases $(3)(a, b)$ of $X=M$ and $G^{\prime}=D i f f^{t}(M)$ these representations can be generalised with the help of the symmetric group $\Sigma_{n}$ representations in the following manner, where $\Sigma_{n}$ is the group of all (bijective) automorphisms $\sigma$ of the set $\{1,2, . ., n\}$ with $n \in \mathbf{N}$ and $\Sigma^{\infty}$ is the symmetric group of $\mathbf{N}$ (that is, of all bijective mappings of $\mathbf{N}$ ). Let $q: \Sigma_{n} \rightarrow U(W)$ be the unitary representation of $\Sigma_{n}$, where $W$ is the Hilbert space, or $q: \Sigma^{\infty} \rightarrow U(W)$. Then $s_{n}: B_{X}^{n} \rightarrow \tilde{X}^{n}$ or $s: \tilde{\Gamma}_{X} \rightarrow \tilde{X}^{\infty}$ produces a mapping $\sigma: G^{\prime} \times B_{X}^{n} \rightarrow$ $\Sigma_{n}$ or $s: G^{\prime} \times \tilde{\Gamma}_{X} \rightarrow \Sigma^{\infty}$ by the formula $s_{n}\left(\psi^{-1}(\gamma)\right)=\psi^{-1}\left(s_{n}(\gamma)\right) \sigma(\psi, \gamma)$ or $s\left(\psi^{-1}(\gamma)\right)=\psi^{-1}(s(\gamma)) \sigma(\psi, \gamma)$, where $s_{n}$ is a measurable cross-section of $p_{n}: \tilde{X}^{n} \rightarrow B_{X}^{n}$ and $s$ of $p: \tilde{X}^{\infty} \rightarrow \tilde{\Gamma}_{X}$ such that $p_{n}\left(x_{1}, \ldots, x_{n}\right)=\left\{x_{1}, \ldots, x_{n}\right\}$ for $n \in \mathbf{N}$ or $p\left(x_{1}, x_{2}, \ldots\right)=\left\{x_{1}, x_{2}, \ldots\right\},\left(x_{1}, \ldots, x_{n}\right) \sigma=\left(x_{\sigma(1)}, \ldots, x_{\sigma(n)}\right)$ or $\left(x_{1}, x_{2}, \ldots\right) \sigma=\left(x_{\sigma(1)}, x_{\sigma(2)}, \ldots\right)$ respectively. Then with each pair $(n, q)$ or $(\infty, q)$ is associated a unitary representation of $G^{\prime}$ in $L^{2}\left(B_{X}^{n}, m^{n}, W\right)$ or in $L^{2}\left(\tilde{\Gamma}_{X}, P_{m}, W\right)$ respectively such that

(ii) $V_{m}^{q}(\psi) f(\gamma):=\rho_{m^{n}}^{1 / 2}(\psi, \gamma) q(\sigma(\psi, \gamma)) f\left(\psi^{-1}(\gamma)\right)$, or

(iii) $V_{m}^{q}(\psi) f(\gamma):=\rho_{P_{m}}^{1 / 2}(\psi, \gamma) q(\sigma(\psi, \gamma)) f\left(\psi^{-1}(\gamma)\right)$,

where $m^{n}$ is the image measure of the direct product of $n$ copies of $m$ by the $\operatorname{map} p_{n}$ and $\rho_{m^{n}}(\psi, \gamma):=\left(m^{n}\right)^{\psi}(d \gamma) / m^{n}(d \gamma),\left(m^{n}\right)^{\psi}(E):=m^{n}\left(\psi^{-1} E\right)$ for each $E \in A f\left(B_{X}^{n}, m^{n}\right), \psi \in G^{\prime}, \gamma \in B_{X}^{n}$. As usually the space $L^{2}\left(B_{X}^{n}, m^{n}, W\right)$ denotes the space of equivalence classes of measurable functions $f: B_{X}^{n} \rightarrow W$ for which $\|f\|_{L^{2}\left(B_{X}^{n}, m^{n}, W\right)}^{2}:=\int_{B_{X}^{n}}\|f(x)\|_{W}^{2} m^{n}(d x)<\infty$. Then $U_{m}$ and $V_{m}^{q}$ 
define new representation $U_{m}^{q}:=U_{m} \otimes V_{m}^{q}$.

3.2. Note. For the group of diffeomorphisms of the real finite-dimensional manifold $M$ such representations were defined in [38], where it was mentioned that the representations $V_{m}^{q}$ are in a weak respect analogous to the construction of $\mathrm{H}$. Weyl for the classical Lie groups. For $W=\{0\}$ and $q=I$ the representation $V_{m}^{q}$ is evidently irreducible [12, 21, 20], hence as in theorem 1.1 [38] for the considered here cases we have, that $(i)$ if $q$ is the irreducible representation of $\Sigma_{n}$ with $n \in \mathbf{N}$, then $V_{m}^{q}$ is the irreducible representation of the diffeomorphism group $G^{\prime} ;(i i) V_{m}^{q_{1}}$ and $V_{m}^{q_{2}}$ are equivalent if and only if $n_{1}=n_{2}$ and $q_{1}$ of $S_{n_{1}}$ is equivalent to $q_{2}$ of $S_{n_{2}}$.

3.3. Note. Let $X=M$ be a finite-dimensional over a local field $\mathbf{K}$ non-compact manifold embedded as an open subset into $\mathbf{K}^{\mathbf{n}}$. Suppose that $m$ is the restriction $m=\left.\lambda\right|_{M}$ of the Haar measure $\lambda$ on $\mathbf{K}^{\mathbf{n}}$ normalised by $\lambda\left(B\left(\mathbf{K}^{\mathbf{n}}, 0,1\right)\right)=1$. Let $\operatorname{Diff}^{t}(X, m)$ denotes the subgroup of $G^{\prime}=\operatorname{Diff}^{t}(X)$ of the non-Archimedean class of smoothness $C^{t}$ such that $\rho_{m}(\psi, x)=1$ for each $\psi \in \operatorname{Diff}^{t}(X, m)$ and $x \in X$, where $1 \leq t \leq \infty$.

3.4. Theorem. Let $X$ and $\operatorname{Diff}^{t}(X, m)$ be the same as in $\S 3.3$. Then the restriction of the representation $V_{m}^{q}$ from $\S 3.1$ on $\operatorname{Diff}^{t}(X, m)$ is irreducible.

Proof. For finite-dimensional $M$ over the local field $\mathbf{L}$ there is the equality $\tilde{\Gamma}_{M}=\Gamma_{M}$ due to $\S 2.4$. Since diffeomorphisms $\psi$ with locally linear $(\psi-i d)$ are contained in $\operatorname{Diff}^{t}(X, m)$, for example, when $\|\psi-i d\|_{C^{1}\left(X \rightarrow \mathbf{K}^{\mathbf{n}}\right)}<1$. Then for each pairwise distinct points $x_{1}, \ldots, x_{n} \in X$ there are neighbourhoods $O_{1}, \ldots, O_{n}$ such that their closures $\bar{O}_{j}$ are $C^{1}$-diffeomorphic with balls in $\mathbf{K}^{\mathbf{n}}$ and $\bar{O}_{i} \cap \bar{O}_{j}=\emptyset$ for each $i \neq j$ and $m\left(O_{1}\right)=\ldots=m\left(O_{n}\right)$. Moreover, for each transposition $\left(k_{1}, \ldots, k_{n}\right)$ of $(1, \ldots, n)$ there exists a diffeomorphism $\psi \in \operatorname{Diff}^{t}(X, m)$ with $\psi\left(\bar{O}_{i}\right)=\bar{O}_{k_{i}}$. Such $\psi$ exists due to partition of $X$ into disjoint union of sufficiently small clopen balls $U_{l}$ such that $\bar{O}_{j}=O_{j}$ for each $j=1, \ldots, n$ and for each $j$ there exists $l_{j}$ such that $O_{j}=U_{l_{j}}$ while $\psi\left(O_{j}\right)=O_{k_{j}}$ and diameters of all $O_{j}$ are equal to each other. Having $\psi$ on $\bigcup_{j=1}^{n} O_{j}=: E$, it is possible to extend $\psi$ as $i d$ on $X \backslash E$.

Let now $Y$ be a clopen compact submanifold of $X$ and consider subspace $\tilde{L}^{2}(Y, m, \mathbf{C})$ consisting of $f \in L^{2}(Y, m, \mathbf{C})$ with $\int_{Y} f(y) m(d y)=0$. Let $H_{1} \neq\{0\}$ be an invariant subspace of $\tilde{L}^{2}(Y, m, \mathbf{C})$ relative to the regular representation $\left(U_{\psi} f\right)(y):=f\left(\psi^{-1} y\right)$ of $\operatorname{Diff}^{t}(Y, m)$. For each ball $O$ in $Y$ there exists $f \in H_{1}$ such that $f \neq 0$ and $\operatorname{supp}(f) \subset O$. Further analogously to the proof of lemma 2 from $\S 1$ [38 we get, that such representation of 
Dif $f^{t}(Y, m)$ is irreducible. If $O_{j}$ with $j=1, \ldots, n$ are clopen balls in $X$, then a subgroup $G^{0}\left(O_{1}, \ldots, O_{n} ; X\right)$ of elements $\psi \in D i f f^{t}(X, m)$ with $\left.\psi\right|_{O_{j}}=i d$ for each $j=1, \ldots, n$ acts trivially on $\left(\otimes_{j=1}^{n} L^{2}\left(O_{j}, m, \mathbf{C}\right)\right) \otimes W$. Then quite analogously to the proof of theorem 1.2 38 we get the statement of this theorem.

3.5. Note. Let $\tilde{\mathbf{N}}^{n}:=\left\{a=\left(i_{1}, \ldots, i_{n}\right) \mid i_{j} \neq i_{s}\right.$ for each $\left.i \neq s\right\}$, $l_{2}\left(\tilde{\mathbf{N}}^{n}, W\right):=\left\{\phi \mid \phi: \tilde{\mathbf{N}}^{n} \rightarrow W\right.$, such that $\left.\|\phi\|^{2}:=\sum_{a \in \tilde{\mathbf{N}}^{n}}\|\phi(a)\|_{W}^{2}<\infty\right\}$ and $H^{q}:=\left\{\phi \in l_{2}\left(\tilde{\mathbf{N}}^{n}, W\right) \mid \phi\left(i_{\sigma(1)}, \ldots, i_{\sigma(n)}\right)=q^{-1}(\sigma) \phi\left(i_{1}, \ldots, i_{n}\right)\right)$ for all $\left.\sigma \in S_{n}\right\}$, where $q$ is a unitary representation of $S_{n}$ in a Hilbert space $W$. In the case $X=M$ the representation $q$ and $W$ may be non-trivial with $n \in \mathbf{N}$, for $X=G$ and $G^{\prime}$ acting on $X$ we set $q=I$ and $W=\{0\}$ and $n=0$. We denote by $\Sigma^{\infty}$ the set of all permutations (bijections) of $\mathbf{N}$ and put $\sigma a=\left(\sigma\left(i_{1}\right), \ldots, \sigma\left(i_{n}\right)\right)$ for $\sigma \in \Sigma^{\infty}$ and $a \in \tilde{\mathbf{N}}^{n}$. Then a function $\sigma: G^{\prime} \times \tilde{\Gamma}_{X} \rightarrow \Sigma^{\infty}$ is defined by the formula $s\left(\psi^{-1}(\gamma)\right)=\psi^{-1}(s(\gamma)) \sigma(\psi, \gamma)$, where $s$ is a measurable (admissible) cross section of the map $p: \tilde{X}^{\infty} \ni$ $\left(x_{1}, x_{2}, \ldots\right) \mapsto\left\{x_{1}, x_{2}, \ldots\right\} \in \tilde{\Gamma}_{X}$ posessing the following property:

$(\alpha)$ if $\operatorname{card}\left(\gamma \cap X_{1}\right)=k_{1}, \operatorname{card}\left(\gamma \cap\left(K_{2} \backslash K_{1}\right)\right)=k_{2}, \ldots, \operatorname{card}\left(\gamma \cap\left(K_{n} \backslash\right.\right.$ $\left.\left.K_{n-1}\right)\right)=k_{n}, \ldots$, then the first $k_{1}$ elements of $s(\gamma)$ are in $\gamma \cap K_{1}$, the next $k_{2}$ of $s(\gamma)$ are in $\gamma \cap\left(K_{2} \backslash K_{1}\right)$ and so on. For $X=M$ finite-dimensional over $\mathbf{L}$ and $\psi \in \operatorname{Diff}^{t}\left(K_{l}\right):=\left\{\psi \in \operatorname{Diff}(X):\left.\psi\right|_{K_{l}^{c}}=i d\right\}$, then $\sigma(\psi, \gamma) \in \Sigma_{r}$, so $H^{q}$ is non-trivial in general for this case, where $K_{l}^{c}:=X \backslash K_{l}$. The latter property in general may be untrue for infinite-dimensional manifold $M$ or for $X=G$ and $G^{\prime}$ acting on $G$, therefore, we consider $q=I$ and $n=0$ and $W=\{0\}$ for $X=G$. For infinite-dimensional $X=M$ over $\mathbf{L}$ let us drop condition $(\alpha)$ and let $q$ be a representation of $\Sigma^{\infty}$ in $U\left(H^{q}\right)$, where $H^{q}$ is defined analogously with the $H^{q}$ for $n$ but with the substitution of $n$ onto $\infty$ and $\Sigma_{n}$ onto $\Sigma^{\infty}$.

Then there exists the following unitary representation of $G^{\prime}$ in the space $L^{2}\left(\tilde{\Gamma}_{X}, P_{m}, \mathbf{C}\right) \otimes H^{q}$ (which is isomorphic with $L^{2}\left(\tilde{\Gamma}_{X}, P_{m}, \mathbf{C}\right)$ for $\left.X=G\right)$ :

(i) $U_{m}^{q}(\psi) F(\gamma, a):=\rho_{P_{m}}^{1 / 2}(\psi, \gamma) F\left(\psi^{-1}(\gamma), \sigma(\psi, \gamma)^{-1} a\right)$ (see $\rho_{P_{m}}$ in $\left.\S 3.1\right)$.

3.6. Theorem. The representations from $\S 3.1$ and $\S 3.5$ in the case $X=G$ are equivalent, in the case of finite-dimensional $X=M$ over $\mathbf{L}$ for the group of diffeomorphism acting on $M$ the representation $U_{m} \otimes V_{m}^{q}$ is equivalent with $U_{n \circ \mathrm{m}}^{q}$, where $q$ is a representation of the symmetric group $\Sigma_{n}$.

Proof. In the case $X=G$ this follows from their definitions, that is, $U_{m}$ given by formula 3.1.( $i$ ) is equivalent with $U_{m}^{q}$, since $q=I$ and $W$ is trivial. 
In the case of the $C^{\infty}$-manifold $X=M$, which is finite-dimensional over $\mathbf{R}$, it was proved in theorem 3.2 38. In the non-Archimedean case the proof is analogous, but instead of differentiabilty of measures their pseudodifferentiability should be considered as in 28]. In view of proposition 2.16 the quasi-invariant measure $m$ on $M$ relative to $\operatorname{Diff}^{t}(M)$ is equivalent with the restriction of the Haar measure $\lambda$ for $\mathbf{L}^{k}$ on $M$, that is, $\left.\lambda\right|_{M} \sim m$, where $M$ is embedded into $\mathbf{L}^{k}$ for the corresponding local field $\mathbf{L}$. In view of $\S 2.4$ $\Gamma_{M}=\tilde{\Gamma}_{M}$ for locally compact $M$. Therefore, convolutions of measures $\mu_{1} * \mu_{2}$ are correctly defined on $\Gamma_{M}$ as an image of the product measure $\mu_{1} \times \mu_{2}$ on $\Gamma_{M} \times \Gamma_{M}$ relative to a mapping $\left(\gamma_{1}, \gamma_{2}\right) \mapsto\left(\gamma_{1} \cup \gamma_{2}\right)$ from $\Gamma_{M} \times \Gamma_{M}$ to $\Gamma_{M}$. Then $n \circ \mu$ denotes $\mu * m_{n}$, where $m_{n}$ is a measure on $B_{X}^{n}$ corresponding to the restriction $\left.P_{m}\right|_{B_{X}^{n}}, n_{1} \circ\left(n_{2} \circ \mu\right)$ is equivalent with $\left(n_{1}+n_{2}\right) \circ \mu$ for each $n_{1}$ and $n_{2} \in \mathbf{N}, 0 \circ \mu$ is equivalent with $\mu$. For each $\psi \in \operatorname{Diff}^{t}(M)$ we have $\psi\left(\mu_{1} * \mu_{2}\right)=\left(\psi \mu_{1}\right) *\left(\psi \mu_{2}\right)$, where $t \geq 1$ and $\psi \mu:=\mu^{\psi}, \mu^{\psi}(E):=\mu\left(\psi^{-1} E\right)$ for each $E \in B f(M)$. Therefore, for each pair $\mu_{1}$ and $\mu_{2}$ of quasi-invariant measures, their convolution is also a quasi-invaraint measure. Then all necessary results from $§ 2.3-5$ of [38] can lightly be transferred onto the nonArchimedean case.

3.7. Note. In the papers [16, 27, 30, 35] quasi-invariant measures on the diffeomorphisms groups of real Banach manifolds were constructed. Purely Gaussian measures quasi-invariant relative to dense subgroups were constructed in the cases of Euclidean and Hilbertian at infinity manifolds and also for definite closed subgroups Diff $f_{k}^{t}(M):=\left\{f \in \operatorname{Diff}^{t}(M)\right.$ : $\left.\left(\Delta^{j} f\right)\right|_{\partial M}=\left.\left(\Delta^{j}\right) i d\right|_{\partial M}$ for each $\left.j=0,1, \ldots, k\right\}$ and $D i f_{l}^{t}(M):=\{f \in$ $\operatorname{Diff}^{t}(M):\left.\left(\partial_{\nu}^{j} f\right)\right|_{\partial M}=\left.\left(\partial_{\nu}^{j} i d\right)\right|_{\partial M}$ for each $\left.j=0,1, \ldots, l\right\}$ of $\operatorname{Diff}^{t}(M)$ for compact $C^{s}$-manifolds $M$ with a boundary $\partial M \neq \emptyset$, where $\operatorname{Diff}^{t}(M)$ has a class of smoothness by Hölder $C^{t}$, also a class of smoothness $H^{t}$ by Sobolev or Besov was considered for $t>\operatorname{dim}_{\mathbf{R}} M+5, \Delta$ denotes the Beltrami-Laplace operator on $M, \partial_{\nu}$ denotes the partial differentiation along normal to the boundary in local coordinates, $\Delta^{0}=I$ and $\partial^{0}=I$ are the unit operators. In particular for a compact manifold with the boundary purely Gaussian measures $\mu$ on $D i f f_{k}^{t}(M)$ and $D i f_{l}^{t}(M)$ quasi-invariant relative to dense subgroups $D i f f_{k}^{t^{\prime}}(M)$ and $D i f_{l}^{t^{\prime \prime}}(M)$ were constructed, where $k$ and $l$ and $t^{\prime}-t>0$ and $t^{\prime \prime}-t>0$ are dependent on $\operatorname{dim}_{\mathbf{R}} M, s>t^{\prime}+2$ and $s>t^{\prime \prime}+2$ respectively. The cases of Schwarz class of smoothness also were considered. The given below theorem in the real case for finite measures was proved shorlty earlier in [25], in the non-Archimedean case it is contained in [18]. 
In theorem 3.8 a quasi-invariant $\sigma$-finite $\sigma$-additive measure is considered, which may be unbounded. The cases of $\sigma$-finite non-negative measures and probability measures on $G$ are considered quite analogously. Certainly this theorem is applicable not only to Gaussian measures but also to measures which have definite properties of the quasi-invariance factors $\rho_{\mu}$ such that a family of continuos functions $\left\{\rho_{\mu}^{1 / 2}(z, g)=\phi(g): z \in G^{\prime}\right\}$ parametrized with $z \in G^{\prime}$ separates points of $G$ (see more precisely the proof below). It is essential in the proof that $G$ is the infinite-dimensional non-locally group and $G^{\prime}$ is its dense subgroup such that the measure $\mu$ is ergodic. Evidently, if $\mu^{\prime}$ is a measure equivalent with $\mu$, then the regular representations associated with them are equivalent due to the isomorphism $f(g) \mapsto\left(\mu^{\prime}(d g) / \mu(d g)\right)^{1 / 2} f(g)$ of the Hilbert space $L^{2}\left(G, \mu^{\prime}, \mathbf{C}\right)$ with $L^{2}(G, \mu, \mathbf{C})$, where $f \in L^{2}\left(G, \mu^{\prime}, \mathbf{C}\right)$ and $g \in G$.

3.8. Theorem. Let $G$ be a group of diffeomorphisms with a real probability quasi-invariant measure $\mu$ relative to a dense subgroup $G^{\prime}$ as in §3.7. Then $\mu$ may be chosen such that the associated regular unitary representation of $G^{\prime}$ is irreducible.

Proof. Let a measure $\nu$ on a Banach space $H$ be of the same type as in the proofs of theorems in papers cited in $\$ 3.7$ such that a local diffeomorphism $A: W \rightarrow V_{H}$ induces a quasi-invaraint measure on $W$ and then with the help of left shifts $g_{j} \in G^{\prime}$ on the entire group $G$, where $W$ is an open neighbourhood of $e$ in $G$ and $V_{H}$ is an open neighbourhood of 0 in $H$. We choose a constant multiplier $c>0$ for $\mu$ such that $c \mu(W)=1$ and then denote such normalized measure by $\mu$. The measure $\mu$ on $G$ is $\sigma$-finite, since $0<\mu(W)<\infty$ and $G$ is with a countable base and a locally finite covering as in $\S 2.8$ and $\S 2.9$. A strong continuity of the regular representation $T: G^{\prime} \rightarrow U\left(L^{2}(G, \mu, \mathbf{C})\right)$ follows from the continuity of the quasiinvariance factor $\rho_{\mu}(\psi, x)$ by $(\psi, x) \in G^{\prime} \times G$ and the embedding $T_{e} G^{\prime} \hookrightarrow T_{e} G$ of trace class, where $T^{\mu}:=T, T(z) f(g):=\rho_{\mu}^{1 / 2}(z, g) f\left(z^{-1} g\right), z \in G^{\prime}, g \in G$, $f \in L^{2}(G, \mu, \mathbf{C})$. Let a $\nu$-measurable function $f: H \rightarrow \mathbf{C}$ be such that $\nu\left(\{x \in H: f(x+y) \neq f(x)\}=0\right.$ for each $y \in X_{0}$ with $f \in L^{1}(H, \nu, \mathbf{C})$. Let also $P_{k}: l_{2} \rightarrow L(k)$ be projectors such that $P_{k}(x)=x_{k}$ for each $x=\left(\sum_{j \in \mathbf{N}} x^{j} e_{j}\right)$, where $x_{k}:=\sum_{j=1}^{k} x^{j} e_{j}, x_{k} \in L(k), L(k):=s p_{\mathbf{R}}\left(e_{1}, \ldots, e_{k}\right)$, $s p_{\mathbf{R}}\left(e_{j}: j \in \mathbf{N}\right):=\left\{y: y \in l_{2} ; y=\sum_{j=1}^{n} x^{j} e_{j} ; x^{j} \in \mathbf{R} ; n \in \mathbf{N}\right\}$. Since the dense subspace $X$ in $H$ is isomorphic with $l_{2}$, then each finite-dimensional subspace $L(k)$ is complemented in $H$ [32]. From the proof of Proposition II.3.1 [5] in view of the Fubini Theorem there exists a sequence of cylindrical 
functions $f_{k}(x)=f_{k}\left(x^{k}\right)=\int_{H \ominus L(k)} f\left(P_{k} x+\left(I-P_{k}\right) y\right) \nu_{I-P_{k}}(d y)$ which converges to $f$ in $L^{1}(H, \nu, \mathbf{C})$, where $\nu=\nu_{L(k)} \otimes \nu_{I-P_{k}}, \nu_{I-P_{k}}$ is the measure on $H \ominus L(k)$. Each cylindrical function $f_{k}$ is $\nu$-almost everywhere constant on $H$, since $L(k) \subset X_{o}$ for each $k \in \mathbf{N}$, consequently, $f$ is $\nu$-almost everywhere constant on $H$. From the construction of $G^{\prime}$ and $\mu$ with the help of the local diffeomorphism $A$ and $\nu$ it follows that, if a function $f \in L^{1}(G, \mu, \mathbf{C})$ satisfies the following condition $f^{h}(g)=f(g)(\bmod \mu)$ by $g \in G$ for each $h \in G^{\prime}$, then $f(x)=$ const $(\bmod \mu)$, where $f^{h}(g):=f(h g), g \in G$.

Let $f(g)=C h_{U}(g)$ be the characteristic function of a subset $U, U \subset G$, $U \in A f(G, \mu)$, then $f(h g)=1 \Leftrightarrow g \in h^{-1} U$. If $f^{h}(g)=f(g)$ is true by $g \in G \mu$-almost everywhere, then $\mu\left(\left\{g \in G: f^{h}(g) \neq f(g)\right\}\right)=0$, that is $\mu\left(\left(h^{-1} U\right) \triangle U\right)=0$, consequently, the measure $\mu$ satisfies the condition $(P)$ from $\oint$ VIII.19.5 [9], where $A \triangle B:=(A \backslash B) \cup(B \backslash A)$ for each $A, B \subset G$. For each subset $E \subset g_{j} W_{j}$ with $g_{j} \in G^{\prime}$ and $W_{j} \subset W$ from $\S 2.9$ the outer measure is bounded, $\mu^{*}(E) \leq 1$, since $\mu(W)=1$ and $\mu$ is non-negative [ [ consequently, there exists $F \in B f(G)$ such that $F \supset E$ and $\mu(F)=\mu^{*}(E)$. This $F$ may be interpreted as the least upper bound in $B f(G)$ relative to the latter equality. In view of the Proposition VIII.19.5 9] the measure $\mu$ is ergodic, that is for each $U \in A f(G, \mu)$ and $F \in A f(G, \mu)$ with $\mu(U) \times \mu(F) \neq$ 0 there exists $h \in G^{\prime}$ such that $\mu((h \circ E) \cap F) \neq 0$.

From Theorem I.1.2 [5] it follows that $(G, B f(G))$ is a Radon space, since $G$ is separable and complete. Therefore, a class of compact subsets approximates from below each measure $\mu^{f}, \mu^{f}(d g):=|f(g)| \mu(d g)$, where $f \in L^{2}(G, \mu, \mathbf{C})=: \bar{H}$. Due to the Egorov Theorem 2.3.7 [8] for each $\epsilon>0$ and for each sequence $f_{n}(g)$ converging to $f(g)$ for $\mu$-almost every $g \in G$, when $n \rightarrow \infty$, there exists a compact subset $\mathrm{K}$ in $G$ such that $\mu(G \backslash \mathrm{K})<\epsilon$ and $f_{n}(g)$ converges on $\mathrm{K}$ uniformly by $g \in \mathrm{K}$, when $n \rightarrow \infty$. In each Hilbert space $L^{2}\left(\mathbf{R}^{\mathbf{n}}, \lambda, \mathbf{R}\right)$ the linear span of functions functions $f(x)=\exp [(b, x)-(a x, x)]$ is dense, where $b$ and $x \in \mathbf{R}^{\mathbf{n}}, a$ is a symmetric positive definite real $n \times n$ matrix, $(*, *)$ is the standard scalar product in $\mathbf{R}^{\mathbf{n}}$ and $\lambda$ is the Lebesgue measure on $\mathbf{R}^{\mathbf{n}}$. If a non-linear operator $U$ on $X$ satisfies conditions of Theorem 26.1 [37], then $\nu^{U}(d x) / \nu(d x)=\left|\operatorname{det} U^{\prime}\left(U^{-1}(x)\right)\right| \rho_{\nu}\left(x-U^{-1}(x), x\right)$, where $\nu^{U}(B):=\nu\left(U^{-1} B\right)$ for each $B \in B f(X), \rho_{\nu}(z, x)=\exp \left\{\sum_{l=1}^{\infty}\left[2\left(z, e_{l}\right)\left(x, e_{l}\right)-\right.\right.$ $\left.\left.\left(z, e_{l}\right)^{2}\right] / \lambda_{l}\right\}$ by Theorem 26.2 [37], where $\lambda_{l}$ and $e_{l}$ are eigenvalues and eigenfunctions of the correlation operator $J$ on $X$ enumerated by $l \in \mathbf{N}, z \in X_{0}$, $\rho_{\nu}(z, x):=\nu_{z}(d x) / \nu(d x), \nu_{z}(B):=\nu(B-z)$ for each $B \in B f(X)$. Since the 
Gaussian measure $\nu$ induces with the help of subalgebras of cylinder subsets in $B f(H)$ and $B f(X)$ the corresponding Gaussian measure on $H$, which is also denoted by $\nu$, then analogous formulas of quasi-invariance factor are true for $\nu$ on $H$ [5]. Hence in view of the Stone-Weierstrass Theorem A.8 [9] an algebra $\mathrm{V}(Q)$ of finite pointwise products of functions from the following space $\operatorname{sp}_{\mathbf{C}}\left\{\psi(g):=(\rho(h, g))^{1 / 2}: h \in G^{\prime}\right\}=: Q$ is dense in $L^{2}(G, \mu, \mathbf{C})$, since $\rho(e, g)=1$ for each $g \in G$ and $L_{h}: G \rightarrow G$ are diffeomorphisms of the manifold $G, L_{h}(g)=h g$.

For each $m \in \mathbf{N}$ there are $C^{\infty}$-curves $\phi_{j}^{b}$, where $j=1, \ldots, m$ and $b \in$ $(-2,2):=\{a: a \in \mathbf{R} ;-2<a<2\}$ is a parameter, such that $\left.\phi_{j}^{b}\right|_{b=0}=e$ and $\left.\left(\partial \phi_{j}^{b} / \partial b\right)\right|_{b=0}$ are linearly independent in $T_{e} G^{\prime}$ vectors and $\phi_{j}:=\phi_{j}^{1}$, $\phi_{j} \in G^{\prime} \cap W, j=1, \ldots, m$, since $G^{\prime}$ is the infinite-dimensional group, which is complete relative to its own uniformity. Then the following condition $\operatorname{det}(\Psi(g))=0$ defines a submanifold $G_{\Psi}$ in $G$ of codimension over $\mathbf{R}$,

(i) $\operatorname{codim}_{\mathbf{R}} G_{\Psi} \geq 1$, where $\Psi(g)$ is a matrix dependent from $g \in G$ with matrix elements $\Psi_{l, j}(g):=D_{\phi_{j}}^{2 l}\left(\rho\left(\phi_{j}, g\right)\right)^{1 / 2}$. If $f \in \bar{H}$ is such that $\left(f(g),(\rho(\phi, g))^{1 / 2}\right)_{\bar{H}}=0$ for each $\phi \in G^{\prime} \cap W$, then differentials of these scalar products by $\phi$ are zero. But $\mathrm{V}(Q)$ is dense in $\bar{H}$ and in view of condition $(i)$ this means that $f=0$, since for each $m$ there are $\phi_{j} \in G^{\prime} \cap W$ such that $\operatorname{det} \Psi(g) \neq 0 \mu$-almost everywhere on $G, g \in G$. If $\|f\|_{\bar{H}}>0$, then $\mu(\operatorname{supp}(f))>0$, consequently, $\mu\left(\left(G^{\prime} \operatorname{supp}(f)\right) \cap W\right)=1$, since $G^{\prime} U=G$ for each open $U$ in $G$ and for each $\epsilon>0$ there exists an open $U, U \supset \operatorname{supp}(f)$, such that $\mu(U \backslash \operatorname{supp}(f))<\epsilon$.

This means that the linear span over C: $s p_{\mathbf{C}}\left\{C h_{g_{k} W_{k}} \phi(g): \phi(g)=\right.$ $\left.\rho_{\mu}^{1 / 2}(h, g) ; h \in G^{\prime}\right\}$ is dense in $L^{2}\left(g_{k} W_{k}, \mu, \mathbf{C}\right)$. Therefore, the following vector $f_{0}(g):=\sum_{j=0}^{\infty} 2^{-j} C h_{g_{j} W_{j}}(g)$ is cyclic for $T^{\mu}$, since $\left\{g_{j} W_{j}: j \in \mathbf{N}_{\mathbf{o}}\right\}$ is a locally finite covering and $\tilde{\mu}(d g)=f_{0}(g) \mu(d g)$ is a finite measure with continuous $\rho_{\tilde{\mu}}$ such that $f(g) \mapsto f_{0}^{1 / 2}(g) f(g)$ establishes isomorphism of $L^{2}(G, \tilde{\mu}, \mathbf{C})$ with $L^{2}(G, \mu, \mathbf{C})$. If $f_{k} \in L^{\infty}\left(g_{k} W_{k}, \mu, \mathbf{C}\right)$ for each $k \in \mathbf{N}$ and $\left.f_{k}\right|_{\left(g_{k} W_{k} \cap g_{l} W_{l}\right)}$ $=\left.f_{l}\right|_{\left(g_{k} W_{k} \cap g_{l} W_{l}\right)}$ for each $g_{k} W_{k} \cap g_{l} W_{l} \neq \emptyset$ and $\sup _{k}\left\|f_{k}\right\|_{L^{\infty}\left(g_{k} W_{k}, \mu, \mathbf{C}\right)}<\infty$, then there exists $f \in L^{\infty}(G, \mu, \mathbf{C})$ such that $\left.f\right|_{g_{k} W_{k}}=f_{k}$ for each $k \in \mathbf{N}$, where $C h_{W}(g)$ is the characteristic function of $W$, that is, $C h_{W}(g)=1$ for each $g \in W$ and $C h_{W}(g)=0$ for each $g \in G \backslash W$. From the construction of $\mu$ it follows that for each $f_{1, j}$ and $f_{2, j} \in \bar{H}, j=1, \ldots, n, n \in \mathbf{N}$ and each $\epsilon>0$ there exists $h \in G^{\prime}$ such that $\left|\left(T_{h} f_{1, j}, f_{2, j}\right)_{\bar{H}}\right| \leq \epsilon\left|\left(f_{1, j}, f_{2, j}\right)_{\bar{H}}\right|$, when $\left|\left(f_{1, j}, f_{2, j}\right)_{\bar{H}}\right|>0$, since $G$ is the Radon space by Theorem I.1.2 [5] and $G$ 
is not locally compact. This means that there is not any finite-dimensional $G^{\prime}$-invariant subspace $H^{\prime}$ in $\bar{H}$ such that $T_{h} H^{\prime} \subset H^{\prime}$ for each $h \in G^{\prime}$ and $H^{\prime} \neq\{0\}$. Hence if there is a $G^{\prime}$-invariant closed subspace $H^{\prime}$ in $\bar{H}$ it is isomorphic with the subspace $L^{2}(V, \mu, \mathbf{C})$, where $V \in B f(G)$.

Let $\mathrm{A}_{G}$ denotes a $*$-subalgebra of $L(\bar{H}, \bar{H})=L(\bar{H})$ generated by the family of unitary operators $\left\{T_{h}: h \in G^{\prime}\right\}$. In view of the von Neumann double commuter Theorem (see §VI.24.2 [9]) $\mathrm{A}_{G}$ " coincides with the weak and strong operator closures of $\mathrm{A}_{G}$ in $L(\bar{H}, \bar{H})$, where $\mathrm{A}_{G}{ }^{\prime}$ denotes the commuting algebra of $\mathrm{A}_{G}$ and $\mathrm{A}_{G}{ }^{\prime \prime}=\left(\mathrm{A}_{G}{ }^{\prime}\right)^{\prime}$. Suppose that $\lambda$ is a probability Radon measure on $G^{\prime}$ such that $\lambda$ has not any atoms and $\operatorname{supp}(\lambda)=G^{\prime}$. Let $a(x) \in$ $L^{\infty}(G, \mu, \mathbf{C}), f$ and $g \in \bar{H}, \beta(h) \in L^{2}\left(G^{\prime}, \lambda, \mathbf{C}\right)$. Since $L^{2}\left(G^{\prime}, \lambda, \mathbf{C}\right)$ is infinitedimensional, then for each finite family of $a \in\left\{a_{1}, \ldots, a_{m}\right\} \subset L^{\infty}(G, \mu, \mathbf{C})$, $f \in\left\{f_{1}, \ldots, f_{m}\right\} \subset \bar{H}$ there exists $\beta(h) \in L^{2}\left(G^{\prime}, \lambda, \mathbf{C}\right), h \in G^{\prime}$, such that $\beta$ is orthogonal to $\int_{G} \bar{f}_{s}(g)\left[f_{j}\left(h^{-1} g\right)(\rho(h, g))^{1 / 2}-f_{j}(g)\right] \mu(d g)$ for each $s, j=$ $1, \ldots, m$. Hence each operator of multiplication on $a_{j}(g)$ belongs to $\mathrm{A}_{G} "$, since there exists $\beta(h)$ such that $\left(f_{s}, a_{j} f_{l}\right)=\int_{G} \int_{G^{\prime}} \bar{f}_{s}(g) \beta(h)(\rho(h, g))^{1 / 2} f_{l}\left(h^{-1} g\right) \lambda(d h) \mu(d g)$ $=\int_{G} \int_{G^{\prime}} \bar{f}_{s}(g) \beta(h)\left(T_{h} f_{l}(g)\right) \lambda(d h) \mu(d g), \int_{G} \bar{f}_{s}(g) a_{j}(g) f_{l}(g) \mu(d g)=\int_{G} \int_{G^{\prime}} \bar{f}_{s}(g)$ $\beta(h) f_{l}(g) \lambda(d h) \mu(d g)=\left(f_{s}, a_{j} f_{l}\right)$. Hence $\mathbf{A}_{G}$ " contains subalgebra of all operators of multiplication on functions from $L^{\infty}(G, \mu, \mathbf{C})$.

Let us remind the following. A Banach bundle B over a Hausdorff space $G^{\prime}$ is a bundle $<B, \pi>$ over $G^{\prime}$, together with operations and norms making each fiber $B_{h}\left(h \in G^{\prime}\right)$ into a Banach space such that conditions $B B(i-i v)$ are satisfied: $B B(i) x \rightarrow\|x\|$ is continuous on $B$ to $\mathbf{R} ; B B(i i)$ the operation + is continuous as a function on $\{(x, y) \in B \times B: \pi(x)=\pi(y)\}$ to $B ; B B(i i i)$ for each $\lambda \in \mathbf{C}$, the map $x \rightarrow \lambda x$ is continuous on $B$ to $B ; B B(i v)$ if $h \in G^{\prime}$ and $\left\{x_{i}\right\}$ is any net of elements of $B$ such that $\left\|x_{i}\right\| \rightarrow 0$ and $\pi\left(x_{i}\right) \rightarrow h$ in $G^{\prime}$, then $x_{i} \rightarrow 0_{h}$ in $B$, where $\pi: B \rightarrow G^{\prime}$ is a bundle projection, $B_{h}:=\pi^{-1}(h)$ is the fiber over $h$ (see $\S I I .13 .4$ [9]). If $G^{\prime}$ is a Hausdorff topological group, then a Banach algebraic bundle over $G^{\prime}$ is a Banach bundle $\mathrm{B}=<B, \pi>$ over $G^{\prime}$ together with a binary operation $\bullet$ on $B$ satisfying conditions $A B(i-v)$ : $A B(i) \pi(b \bullet c)=\pi(b) \pi(c)$ for $b$ and $c \in B ; A B(i i)$ for each $x$ and $y \in G^{\prime}$ the product $\bullet$ is bilinear on $B_{x} \times B_{y}$ to $B_{x y} ; A B(i i i)$ the product $\bullet$ on $B$ is associative; $A B(i v)\|b \bullet c\| \leq\|b\| \times\|c\|(b, c \in B) ; A B(v)$ the map $\bullet$ is continuous on $B \times B$ to $B$ (see $\S$ VIII.2.2 [9]). With $G^{\prime}$ and a Banach algebra A the trivial Banach bundle $\mathrm{B}=\mathrm{A} \times G^{\prime}$ is associative, in particular let $\mathrm{A}=\mathrm{C}$ (see §VIII.2.7 [G]).

The regular representation $T$ of $G^{\prime}$ gives rise to a canonical regular $\bar{H}$ - 
projection-valued measure $\bar{P}: \bar{P}(W) f=C h_{W} f$, where $f \in \bar{H}, W \in B f(G)$, $C h_{W}$ is the characteristic function of $W$. Therefore, $T_{h} \bar{P}(W)=\bar{P}(h \circ W) T_{h}$ for each $h \in G^{\prime}$ and $W \in B f(G)$, since $\rho\left(h, h^{-1} \circ g\right) \rho(h, g)=1=\rho(e, g)$ for each $(h, g) \in G^{\prime} \times G, C h_{W}\left(h^{-1} \circ g\right)=C h_{h \circ W}(g)$ and $T_{h}(\bar{P}(W) f(g))=$ $\rho(h, g)^{1 / 2} \bar{P}(h \circ W) f\left(h^{-1} \circ g\right)$. Thus $\langle T, \bar{P}\rangle$ is a system of imprimitivity for $G^{\prime}$ over $G$, which is denoted $\mathrm{T}^{\mu}$. This means that conditions $S I(i-i i i)$ are satisfied: $S I(i) T$ is a unitary representation of $G^{\prime} ; S I(i i) \bar{P}$ is a regular $\bar{H}$-projection-valued Borel measure on $G$ and $S I(i i i) T_{h} \bar{P}(W)=\bar{P}(h \circ W) T_{h}$ for all $h \in G^{\prime}$ and $W \in B f(G)$.

For each $F \in L^{\infty}(G, \mu, \mathbf{C})$ let $\bar{\alpha}_{F}$ be the operator in $L(\bar{H}, \bar{H})=L(\bar{H})$ consisting of multiplication by $F: \bar{\alpha}_{F}(f)=F f, f \in \bar{H}$. The map $F \rightarrow \bar{\alpha}_{F}$ is an isometric $*$-isomorphism of $L^{\infty}(G, \mu, \mathbf{C}$ ) into $L(\bar{H}, \bar{H})$ (see $\S$ VIII.19.2 [9]). Therefore, Propositions VIII.19.2,5 9] (using the approach of this particular case given above) are applicable in our situation.

If $\bar{p}$ is a projection onto a closed $\mathrm{T}^{\mu}$-stable subspace of $\bar{H}$, then $\bar{p}$ commutes with all $\bar{P}(W)$. Hence $\bar{p}$ commutes with multiplication by all $F \in$ $L^{\infty}(G, \mu, \mathbf{C})$, so by VIII.19.2 [9] $\bar{p}=\bar{P}(V)$, where $V \in B f(G)$. Also $\bar{p}$ commutes with all $T_{h}, h \in G^{\prime}$, consequently, $(h \circ V) \backslash V$ and $\left(h^{-1} \circ V\right) \backslash V$ are $\mu$-null for each $h \in G^{\prime}$, hence $\mu((h \circ V) \triangle V)=0$ for all $h \in G^{\prime}$. In view of ergodicity of $\mu$ and proposition VIII.19.5 [9] either $\mu(V)=0$ or $\mu(G \backslash V)=0$, hence either $\bar{p}=0$ or $\bar{p}=I$, where $I$ is the unit operator. Hence $T$ is the irreducible unitary representation.

Almost analogous proof was done in the case of loop groups with the corresponding quasi-invaraint measures and with the use of the spectral theorem for the family of commuting unitary operators, since the loop group is Abelian in [23, 24]. In the non-Archimedean case $G^{\prime}$ has the analytic atlas $A t\left(G^{\prime}\right)=\left\{\left(U_{j}, \psi_{j}\right): j \in \mathbf{N}\right\}$ with disjoint clopen charts, hence curves $\phi_{j}^{b}$ can be chosen locally analytic with a restriction on the corresponding neighbourhood $U_{1}$ of $e$ being analytic, where $b \in \mathbf{L}$. Substitution of differentiation on pseudodifferentiation along $\phi_{j}^{b}$ by parameter $b \in B(\mathbf{L}, 0,1)$ produces by formula $\operatorname{det}(\Psi(g))=0$ an analytic submanifold $G_{\Psi}$ in $G$ with $\operatorname{codim}_{\mathbf{L}} G_{\Psi} \geq 1$, since $G$ is the analytic manifold.

3.9. Theorem. Let $P_{m}$ be the ergodic Poisson measure on $\tilde{\Gamma}_{X}$ as in §2.4, 2.9 and $q$ be an irreducible representation of the symmetric group $\Sigma_{n}$ $(q=I$ for $X=G$ and may be non-trivial for $X=M$ finite-dimensional over the coresponding field $\mathbf{L}$ and a group of diffeomorphisms $G^{\prime}$ of $\left.M\right)$. Then the representation $U_{m} \otimes V_{m}^{q}$ from $§ 3.1$ is irreducible. 
Proof. The case of real finite-dimensional $M$ was proved in [38]. The case of non-Archimedean $M$ with $\operatorname{dim}_{\mathbf{L}} M<\infty$ follows from $\S 3.5$, since $\tilde{\Gamma}_{X}=\Gamma_{X}$ in this case. Indeed, $U_{m} \otimes V_{m}^{q}$ is equivalent with $U_{n \circ m}^{q}$ and $f \phi \in$ $L^{2}\left(\Gamma_{X}, P_{m}, \mathbf{C}\right)$, if $f \in L^{2}\left(\Gamma_{X}, P_{m}, \mathbf{C}\right)$ and $\phi \in L^{\infty}\left(\Gamma_{X}, P_{m}, \mathbf{C}\right)$. Then each subspace $\mathrm{L}$ in $L^{2}\left(\Gamma_{X}, P_{m}, \mathbf{C}\right) \otimes H^{q}$ invariant relative to $G^{\prime}=\operatorname{Diff} f^{t}(X)$ is also invariant relative to multiplications on functions $\phi \in L^{\infty}\left(\Gamma_{X}, P_{m}, \mathbf{C}\right)$, since $\mathrm{L}=\bigoplus_{r, i} \mathrm{~L}_{l, r}^{i}$, where $\mathrm{L}_{l, r}^{i}:=\mathrm{L} \cap\left(L^{2}\left(B_{K_{l}}^{r} \times \Gamma_{X \backslash K_{l}}, \mu_{r}, \mathbf{C}\right) \otimes W_{r}^{i} \otimes C_{r}^{i}\right)$ are subspaces invariant relative to $\operatorname{Diff} f^{t}\left(K_{l}\right), \mu=P_{m}$ and $\mu_{r}$ is the corresponding measure on $B_{K_{l}}^{r} \times \Gamma_{X \backslash K_{L}}$. In view of lemma 2.14 the measure $\mu_{n}$ is equivalent with $\mu_{n}^{\prime} \times \mu_{n}^{\prime \prime}$ and further as at the end of $\S 3$ [38].

The remaining cases are proved analogously to the proof of theorem 3.8 (and see [18, 23, 24]) applied to the pair $\left(G^{\prime}, \tilde{\Gamma}_{X}\right)$ instead of $\left(G^{\prime}, G\right)$, since $\tilde{\Gamma}_{X}$ is $C^{\infty}$-manifold and from infinite differentiability or pseudodifferentiability of $m$ it follows, that $P_{m}$ is also infinite differentiable or pseudodifferentiable respectively, morever, $P_{m}$ is the ergodic measure due to theorem 2.9 . In the case of $X=M$ the measures on $X$ are chosen to be such that $\operatorname{sp}_{\mathbf{C}}\left\{\rho_{m}^{1 / 2}(z, x)=\right.$ $\left.\phi(x): z \in G^{\prime}\right\}$ is dense in $L^{2}(X, m, \mathbf{C})$ in accordance with $\S 2.9$ and the cited papers there, for example, Gaussian measures or product measures of special type on $T_{x} M$ induce the demanded measures on $M$, where $x \in M$.

It remains only to establish that the density $\rho_{P_{m}}$ has the demanded properties. For this it is necessary to use the fact that operators $L_{h}$ on $X$ (either $X=M$ or $X=G$ ) are infinitely strongly differentiable by $h \in G^{\prime}$ and there exists a dense subset $G$ " in $G^{\prime}$ such that $\left(L_{h}\right)^{(n)} \neq 0$ for each $n \in \mathbf{N}$ and each $h \in G^{\prime \prime}$. Therefore, $\left(A L_{h} A^{-1}\right)^{(n)} \neq 0$ for each $h \in G^{\prime \prime}$, where $A: U \rightarrow V_{H}$ is a local diffeomorphism, where $U$ and $V_{H}$ are open subsets in $X$ and the corresponding Banach space $H$ respectively as in $\S 2.9, \S 3.8$ and the cited above papers. In the Hilbert space $L^{2}\left(\mathbf{R}^{\mathbf{m n}}, \lambda, \mathbf{C}\right)$ is dense the following linear span $\operatorname{sp}_{\mathbf{C}}\left\{\exp \left[\sum_{l=1}^{k}\left(a_{1}^{l}, x^{l}\right)-\left(a_{2}^{l} x^{l}, x^{l}\right)\right]=: \phi(y) \mid a_{1}^{l} \in \mathbf{R}^{\mathbf{m}}\right.$, $a_{2}^{l} \in \mathbf{R}^{\mathbf{m}}, \sum_{l=1}^{k} a_{2, j}^{l}>0$ for each $j=1, \ldots, m ; a_{2, j}^{l} \geq 0$ for each $j=1, \ldots, m$, $l=1, \ldots, k$; and if $a_{1, j}^{l} \neq 0$, then there exists $l^{\prime}$ such that $2 l^{\prime}>l$ and $a_{2, j}^{l^{\prime}}>0$, $\left.a_{i}^{l}:=\left(a_{1, i}^{l}, \ldots, a_{i, m}^{l}\right), a_{i, j}^{l} \in \mathbf{R}, x_{j}^{l}:=S_{l}\left(y_{j, 1}, \ldots, y_{j, n}\right), l=1, \ldots, k, j=1, \ldots, m\right\}$, where $k=k(m, n) \in \mathbf{N}$ is chosen such that $z \mapsto\left\{S_{l}(z): l=1, . ., k\right\}$ is a bijection of $\mathbf{R}^{\mathbf{n}}, z=\left(z_{1}, \ldots, z_{n}\right) \in \mathbf{R}^{\mathbf{n}}, S_{l}(z):=\sum_{i=1}^{n}\left(z_{i}\right)^{l}$ is a power sum of degree $l,(*, *)$ is a scalar product in $\mathbf{R}^{\mathbf{m}}, \lambda_{m n}$ is a Lebsgue measure on $\mathbf{R}^{\mathbf{m n}}$. For the local field $\mathbf{L}$ in the Hilbert space $L^{2}\left(\mathbf{L}^{\mathbf{m n}}, \nu, \mathbf{C}\right)$ is dense the following linear $\operatorname{span} \operatorname{sp}_{\mathbf{C}}\left\{\exp \left[\sum_{l=1}^{k}-\left|\left(a^{l},\left.\left(b^{l}+x^{l}\right)\right|^{2}\right]=: \phi(y)\right| a^{l} \in \mathbf{L}^{\mathbf{m}}, b^{l} \in \mathbf{L}^{\mathbf{m}}, \sum_{l=1}^{k}\left|a_{j}^{l}\right|>0\right.\right.$ 
for each $j=1, \ldots, m, a^{l}:=\left(a_{1}^{l}, \ldots, a_{m}^{l}\right), a_{j}^{l} \in \mathbf{L}, x_{j}^{l}:=S_{l}\left(y_{j, 1}, \ldots, y_{j, n}\right)$, $l=1, \ldots, k, j=1, \ldots, m\}$, where $k=k(m, n) \in \mathbf{N}$ is chosen such that $z \mapsto\left\{S_{l}(z): l=1, \ldots, k\right\}$ is a bijection of $\mathbf{L}^{\mathbf{n}}, z=\left(z_{1}, \ldots, z_{n}\right) \in \mathbf{L}^{\mathbf{n}}$, $S_{l}(z):=\sum_{i=1}^{n}\left(z_{i}\right)^{l}$ is a power sum of degree $l,(z, q):=\sum_{i=1}^{n} z_{i} q_{i}, q \in \mathbf{L}^{\mathbf{n}}$, $\nu_{m n}$ is the Haar measure on $\mathbf{L}^{\mathbf{m n}}$.

Using charts in $B_{X}^{n}$ we get projections $L^{2}\left(B_{X}^{n}, m^{n}, \mathbf{C}\right)$ into $L^{2}\left(\mathbf{R}^{\mathbf{m n}}, \lambda_{m n}, \mathbf{C}\right)$ in the real case and into $L^{2}\left(\mathbf{L}^{\mathbf{m n}}, \nu_{m n}, \mathbf{C}\right)$ in the non-Archimedean case. Then we use Taylor expansion up to $o\left(d_{G^{\prime}}^{k^{\prime}+1}(\psi, h)\right)$ of $L_{h}$ in a suitable neighbourhoods $h U^{\prime}$ in $G^{\prime}$ of elements $h \in G^{\prime \prime}$ with $U^{\prime}$ open in $G^{\prime}$ with $e \in U^{\prime} \subset W^{\prime}$ and with $k^{\prime}=2 k$ in the real case and $k^{\prime}=k$ in the non-Archimedean case, where $d_{G^{\prime}}$ is the metric in $G^{\prime}$ in its own uniformity, $\psi \in h U^{\prime}$. For a manifold $C^{t}(M, N)$ of mappings $f: M \rightarrow \mathbf{N}$ of class of smoothness $C^{t}$ with $t \geq 1$ for $C^{\infty}$-manifolds $M$ and $N$ in the real case and analytic manifolds in the non-Archimedean case the tangent manifold $T C^{t}(M, N)$ is isomorphic with $C^{t}(M, T N)$ and for the $n$-th order we get $T^{n} C^{t}(M, N)=$ $C^{t}\left(M, T^{n} N\right)$ (see also [6, 13, 24]). Then $T^{n} \operatorname{Diff}^{t}(M)$ is a submanifold in $C^{t}\left(M, T^{n} M\right)$. Let $C^{t}\left(M, m_{0} ; N, n_{0}\right)$ be a family of mappings $f \in C^{t}(M, N)$ preserving marked points $f\left(m_{0}\right)=n_{0}, m_{0} \in M$ and $n_{0} \in N$, where in the real case $M=S^{m}$ is the $m$-dimensional real sphere and $\operatorname{dim}_{\mathbf{R}} N>m$. Analogously for others classes of smoothness $\omega$ considered for construction of loop groups $L\left(M, m_{0} ; N, n_{0}\right)_{\omega}$, elements of which are closures of orbits $c l\left\{f(\psi(x)): \psi \in G(M), \psi\left(m_{0}\right)=m_{0}\right\}$, where $G(M)$ denotes the group of diffeomorphisms of $M$ of the corresponding class of smoothness and with certain additional construction in the non-Archimedean case [19, 23, 24. Hence the manifold $T^{n} L\left(M, m_{0} ; N, n_{0}\right)_{\omega}$ is isomorphic with the following manifold $T^{n} L\left(M, m_{0} ; T^{n} N,\left(n_{0}, \overline{0}_{n}\right)\right)_{\omega} \otimes T_{\left(n_{0}, \overline{0}_{n}\right)}^{n} N$, where $\overline{0}_{n} \in T^{n} N$ is the zero section for each $n \in \mathbf{N}$. Therefore, it is possible to vary values of differentials $D^{j} f$ for $j=0, \ldots, n$ in the notation $T^{n} f:=\left(f, D f, D^{2} f, \ldots, D^{n} f\right)$ with $D^{0} f:=f$ for elements $f \in G$ " both in the case of the diffeomorphism group and the loop group up to the corrections $o\left(d_{G^{\prime}}^{n+1}(f, \psi)\right)$. Then $D_{h}^{n} L_{h}(g)$ can be expressed through $D^{j} h$ and $D^{j} g$ with $1 \leq j \leq n$, where $h, g \in G$, hence it is possible to vary coefficients $a_{1}^{l}, a_{2}^{l}$ in the real case and $a^{l}, b^{l}$ in the non-Archimedean case.

Take for example, the Gaussian measure on $X$ in the real case induced from the Gaussian measure on the corresponding Banach space and given with the help of non-degenerate symmetric positive definite operator of trace 
class. In the non-Archimedean case each Banach space over a local field $\mathbf{L}$ is isomorphic with $c_{0}(\alpha, \mathbf{L})$, where $\alpha$ is an ordinal and elements of $c_{0}(\alpha, \mathbf{L})$ have the form $x=\left(x_{j}: j \in \alpha, x_{j} \in \mathbf{L}\right)$ such that $\|x\|:=\sup _{j}\left|x_{j}\right|<\infty$ and for each $\epsilon>0$ a set $\left\{j:\left|x_{j}\right|>\epsilon\right\}$ is finite [34]. For each separable manifold $M$ we have $\operatorname{card}(\alpha) \leq \aleph_{0}$. In the latter case take, for example, the following non-Archimedean analog $\eta$ of the Gaussian measure: each projection $\eta_{j}$ of $\eta$ on $\mathbf{L} e_{j}$ has a density $\eta_{j}(d x)=F_{j} \exp \left(-|x|^{2} s_{j}\right) v(d x)$, where $\sum_{j} s_{j}^{-1}<\infty$, $e_{j}:=(0, \ldots, 0,1,0 \ldots)$ with 1 on the $j$-th place, $v$ is the Haar measure on $\mathbf{L}$ with $v(B(\mathbf{L}, 0,1))=1$ and constants $F_{j}>0$ are chosen such that $\eta_{j}(\mathbf{L})=1$ (see also $\S 2.9$ ).

Let $\psi_{j}^{b}$ be $C^{\infty}$-curves in $G^{\prime}$ such that $\left.\left(\partial \psi_{j}^{b} / \partial b\right)\right|_{b=0}$ are linearly independent vectors in $T_{g_{k}} G^{\prime}$ and $\left.\psi_{j}^{b}\right|_{b=0}=g_{k}$ and $R\left(\psi_{j}\right) \cap R\left(\psi_{l}\right) \cap\left(g_{k} W_{k}\right)=\left\{g_{k}\right\}$ for each $j \neq l, b \in \mathbf{L}$, where either $X=M$ or $X=G, j=1, \ldots, n, n \in \mathbf{N}, R(\psi)$ denotes the range of $\psi$, that is, $R(\psi):=\left\{\psi^{b}: b \in \mathbf{L}\right\}, g_{k} \in G^{\prime}, S_{k}$ is open in $\tilde{\Gamma}_{X}, \zeta_{k}: S_{k} \rightarrow Q_{k}$ are local diffeomorphisms of open subsets $S_{k}$ in $\tilde{\Gamma}_{X}$ and $\Gamma_{X}$ respectively, $\gamma \in g_{k} S_{k}$ (see also $\S 2.4$ ).

There are embeddings $L^{2}\left(B_{K}^{n}, m_{n}, \mathbf{C}\right) \hookrightarrow L^{2}\left(B_{X}^{n}, m_{n}, \mathbf{C}\right) \hookrightarrow L^{2}\left(\Gamma_{X}, P_{m}, \mathbf{C}\right) \hookrightarrow$ $L^{2}\left(\tilde{\Gamma}_{X}, P_{m}, \mathbf{C}\right)$, where $m_{n}$ denotes the restriction of $P_{m}$ on $B_{X}^{n}$. For each $x \in X$ there exists $K \in\left\{K_{l}: l \in \mathbf{N}\right\}$ such that $x \in \operatorname{Int}(K)$. Then $\Gamma_{K}$ is the disjoint union of $\left\{B_{K}^{n}: n \in \mathbf{N}_{\mathbf{o}}\right\}$. On the other hand, $\left.P_{K, m}\right|_{B f\left(K^{n}\right)}=m_{K, n}$ in accordance with $\S 3.1$, where $m_{K, n}$ is equivalent with $m_{K}^{n}$, where $m_{K}$ denotes the restriction of $m$ on $B f(K)$ and $m_{K}^{n}$ is the product of $n$ copies of $m_{K}$. Then the condition $\operatorname{det}(\Psi(\gamma))=0$ defines a submanifold $\tilde{\Gamma}_{X, \Psi}$ in $\tilde{\Gamma}_{X}$ of codimension over $\mathbf{L}$,

(i) $\operatorname{codim}_{\mathbf{L}} \tilde{\Gamma}_{X, \Psi} \geq 1$, where $\Psi(\gamma)$ is a matrix dependent from $\gamma \in \tilde{\Gamma}_{X}$ with indices of rows and columns $j$ and $l=1, \ldots, n$ for $n \in \mathbf{N}$ with matrix elements $\Psi_{l, j}(\gamma):=D_{\phi_{j}}^{2 l}\left(\rho\left(\phi_{j}, g\right)\right)^{1 / 2}$ in the real case and with the corresponding pseudodifferentials by parameters $b_{j} \in B(\mathbf{L}, 0,1)$ and for $\phi_{j}^{b_{j}}$ in the non-Archimedean case instead of differentials (see also [18, 28]). In the nonArchimedean case $\tilde{\Gamma}_{X}$ has the analytic atlas $\operatorname{At}\left(\tilde{\Gamma}_{X}\right)=\left\{\left(V_{j}, \omega_{j}\right): j \in \mathbf{N}\right\}$ with disjoint clopen charts, also $G^{\prime}$ has disjoint clopen charts and the analytic atlas $A t\left(G^{\prime}\right)=\left\{\left(U_{j}, \phi_{j}\right): j\right\}$, hence curves $\psi_{j}^{b}$ can be chosen locally analytic with a restriction on the corresponding neighbourhood $U_{1}$ of $e$ in $G^{\prime}$ being analytic, where $b \in \mathbf{L}$. Substitution of differentiation on pseudodifferentiation along $\phi_{j}^{b}$ by parameter $b \in B(\mathbf{L}, 0,1)$ produces by formula $\operatorname{det}(\Psi(\gamma))=0$ an analytic submanifold $\tilde{\Gamma}_{X, \Psi}$ in $\tilde{\Gamma}_{X}$ with $\operatorname{codim}_{\mathbf{L}} \tilde{\Gamma}_{X, \Psi} \geq 1$. 
Since for equivalent measures such regular representations are equivalent, we can consider infinitely differentiable or pseudodifferentiable measures in the real and non-Archimedean cases respectively. There is the following equality $\lim _{\left(m^{n}(B) \rightarrow 0, \infty>m^{n}(B)>0\right)} m^{n}(B) \exp \left(-m^{n}(B)\right) /\left(\left(m^{n}\right)^{\psi}(B) \exp \left(-\left(m^{n}\right)^{\psi}(B)\right)\right)=$ : $m^{n}(d x) \exp \left(-m^{n}(d x)\right) /\left(\left(m^{n}\right)^{\psi}(d x) \exp \left(-\left(m^{n}\right)^{\psi}(d x)\right)\right)=\rho_{m^{n}}(\psi, x)$, since $\rho_{m^{n}}(\psi, x)$ is continuous on $G^{\prime} \times \tilde{X}^{n}$ and $\lim _{\left(m^{n}(B) \rightarrow 0, \infty>m^{n}(B)>0, x \in B\right)} \exp \left(-m^{n}(B)[1-\right.$ $\left.\left.\int_{B} \rho_{m^{n}}(\psi, y) m^{n}(d y) / m^{n}(B)\right]\right)=1$ for balls $B$ in $\tilde{X}^{n}$ such that $x \in \operatorname{Int}(B)$, where $\left(m^{n}\right)^{\psi}(E):=m^{n}\left(\psi^{-1} E\right)$ for each Borel subset $E \in B f\left(\tilde{X}^{n}\right), \psi \in G^{\prime}$, $x \in \tilde{X}^{n}$.

In the case $X=G$ each space $L^{2}\left(B_{X}^{r}, m_{r}, \mathbf{C}\right)$ has the embedding into $L^{2}\left(\tilde{\Gamma}_{X}, P_{m}, \mathbf{C}\right)$, where $m_{r}:=\left.P_{m}\right|_{B_{X}^{r}}$. It was supposed above that the quasiinvariance factor $\rho_{m}(\psi, x)$ of the quasi-invariant measure $m$ on $B f(X)$ relative to $G^{\prime}$ is continuous on $G^{\prime} \times X$, consequently, $\rho_{m^{r}}(\psi, \eta)$ and $\rho_{m_{r}}\left(\psi, \gamma^{r}\right)$ and $\rho_{P_{m}}(\psi, \gamma)$ are continuous on $G^{\prime} \times X^{r}$ and $G^{\prime} \times B_{X}^{r}$ and $G^{\prime} \times \Gamma_{X}$ respectively, where $\psi \in G^{\prime}, \eta \in X^{r}, \gamma^{r} \in B_{X}^{r}, \gamma \in \Gamma_{X}$. Hence due to the definiton of $P_{m}$ there is the equality: $\lim _{r \geq n, r \rightarrow \infty} \rho_{m_{r}}\left(\psi, \gamma^{n}\right)=\rho_{P_{m}}\left(\psi, \gamma^{n}\right)$ for each $\psi \in G^{\prime}$, $\gamma^{n} \in B_{X}^{n}$. If $f \in \bar{H}:=L^{2}\left(\tilde{\Gamma}_{X}, P_{m}, \mathbf{C}\right)$ is such that $\left(f(g),\left(\rho_{P_{m}}(\phi, g)\right)^{1 / 2}\right)_{\bar{H}}=0$ for each $\phi \in G^{\prime} \cap W$, then differentials of these scalar products by $\phi$ are zero. In view of the above embeddings and formula $2.4(i)$ and in view of condition (i) this means that $f=0$, since for each $n \in \mathbf{N}$ there are $\phi_{j} \in G^{\prime} \cap W$ such that $\operatorname{det} \Psi(\gamma) \neq 0 P_{m}$-almost everywhere on $\tilde{\Gamma}_{X}, \gamma \in \tilde{\Gamma}_{X}$. If $\|f\|_{\bar{H}}>0$, then $P_{m}(\operatorname{supp}(f))>0$, consequently, $P_{m}\left(G^{\prime} \operatorname{supp}(f)\right)=1$, since $G^{\prime} U=\tilde{\Gamma}_{X}$ for each open $U$ in $\tilde{\Gamma}_{X}$ and for each $\epsilon>0$ there exists an open $U, U \supset \operatorname{supp}(f)$, such that $P_{m}(U \backslash \operatorname{supp}(f))<\epsilon$.

This means that the linear span over C: $s p_{\mathbf{C}}\left\{C h_{g_{k} S_{k}} \phi(g): \phi(g)=\right.$ $\left.\rho_{P_{m}}^{1 / 2}(h, g) ; h \in G^{\prime}\right\}$ is dense in $L^{2}\left(g_{k} S_{k}, P_{m}, \mathbf{C}\right)$, since $U^{\prime}{ }_{l} K_{l} \subset \operatorname{Int}\left(K_{l+1}\right)$ for each $l \in \mathbf{N}$ (see $\S 2.9$ ). Therefore, $\operatorname{sp}_{\mathbf{C}}\left\{\phi(g): \phi(g)=\rho_{P_{m}}^{1 / 2}(h, g) ; h \in G^{\prime}\right\}$ is dense in $L^{2}\left(\tilde{\Gamma}_{X}, P_{m}, \mathbf{C}\right)$ and a vector $f_{0}$ is cyclic for $U_{m}$, where $f_{0}(\gamma)=1$ for each $\gamma \in \tilde{\Gamma}_{X}$. Then $\mathrm{A}_{G}$ " contains subalgebra of all operators of multiplication on functions from $L^{\infty}\left(\tilde{\Gamma}_{X}, P_{m}, \mathbf{C}\right)$ and the remainder of the proof of theorem 3.9 is quite analogous with the proof of theorem 3.8 (certainly $\mathrm{A}_{G} " \neq L^{\infty}\left(\tilde{\Gamma}_{X}, P_{m}, \mathbf{C}\right) I$ for $G^{\prime}=\operatorname{Diff}^{t}(M)$, since the regular representation $U_{m}(h)$ of $G^{\prime}$ contains a family of cardinality c $:=\operatorname{card}(\mathbf{R})$ of non-commuting operators from the set $\left.\left\{U_{m}(h): h \in G^{\prime}\right\}\right)$.

3.10. Theorem. $(\alpha)$. If there exists a bounded operator $T: L^{2}\left(\tilde{\Gamma}_{X}, P_{m}, \mathbf{C}\right) \otimes$ $H^{q} \rightarrow L^{2}\left(\tilde{\Gamma}_{X}, P_{m^{\prime}}, \mathbf{C}\right) \otimes H^{q^{\prime}}\left(H^{q}=\{0\}\right.$ and $P_{m}$ is from theorem 3.9 for 
$X=G$ or infinite-dimensional $X=M$ over the corresponding field $\mathbf{L}$ such that $\left.L^{2} \otimes\{0\}:=L^{2}\right)$ satisfying conditions $(a, b)$ :

(a) $T U_{m}^{q}(\psi)=U_{m^{\prime}}^{q^{\prime}} T$ for all $\psi \in G^{\prime}$,

(b) there exists $\phi \in H^{q}$ such that $T(1 \otimes \phi) \neq 0$,

then $P_{m}$ and $P_{m^{\prime}}$ are equivalent.

$(\beta)$. If there exists a bounded operator $V: L^{2}(G, \mu, \mathbf{C}) \rightarrow L^{2}\left(G, \mu^{\prime}, \mathbf{C}\right)$ such that $V T^{\mu}(\psi)=T^{\mu^{\prime}}(\psi) V$ for each $\psi \in G^{\prime}$, where $\mu$ is a quasi-invaraint measure on $G$ relative to $G^{\prime}$ and $T^{\mu}$ is the associated regular representation of $G^{\prime}$ from theorem 3.8, then $\mu$ and $\mu^{\prime}$ are equivalent.

The proof is divided into several parts. At first the case $(\alpha)$ of $X=M$ finite-dimensional over the corresponding filed $\mathbf{L}$ is considered in subparagraphs I-III. The cases $(\alpha)$ of $X=G$ and infinite-dimensional $X=M$ over $\mathbf{L}$ and the cases $(\beta)$ are considered in $\S 3.10 . \mathbf{I V}$.

I. Suppose that $\|\phi\|=1$ and $T$ is a contraction operator. Take $X_{n}:=K_{n}$, where $n \in \mathbf{N}$ and $K_{n}$ are the same as in $\S 2.9$. In the case $X=M$ we put $Y=X_{n}, \mu=P_{m}, \mu^{\prime}=P_{m^{\prime}}, \mu_{1}$ and $\mu_{2}$ are equal to the image measure of $\mu$ in accordance with the maps: $\gamma \mapsto(\gamma \cap Y)=: \gamma_{1}, \gamma \mapsto\left(\gamma \cap Y^{c}\right)=: \gamma_{2}$. Apart from the case $X=M$, for $X=G$ we suppose that $Y=X$, since $G^{\prime}$ acts on $G$ transitively and $\operatorname{supp}\left(L_{\psi}\right):=\operatorname{cl}\{g \in G: \psi g \neq g\}=G$ for each $\psi \neq e$, because $G^{\prime}$ is a dense subgroup of $G$ and from $h g=g$ it follows $h=e$, where $h, g \in G$. In the case of $\operatorname{Diff}^{t}(X)$ there exists a bounded operator $T_{Y}: L^{2}\left(\Gamma_{Y}, \mu_{1}, \mathbf{C}\right) \otimes H^{q} \rightarrow L^{2}\left(\Gamma_{Y}, \mu_{1}^{\prime}, \mathbf{C}\right) \otimes H^{q^{\prime}}$ such that

(i) $T_{Y} F\left(\gamma, a^{\prime}\right)=\int_{\Gamma_{Y^{c}}} T F\left(\gamma_{1}, \gamma_{2}, a^{\prime}\right) \mu_{2}^{\prime}\left(d \gamma_{2}\right)$. Then $L^{2}\left(\Gamma_{Y}, \mu_{1}, \mathbf{C}\right)$ is embeddable as a closed subspace into $L^{2}\left(\Gamma_{X}, \mu, \mathbf{C}\right)$ by the map $L^{2}\left(\Gamma_{Y}, \mu_{1}, \mathbf{C}\right) \ni$ $f(\gamma) \mapsto \hat{f}(\gamma):=f(\gamma \cap Y) \in L^{2}\left(\Gamma_{X}, \mu, \mathbf{C}\right)$. Therefore, $T_{Y} F$ depends on $\left(\gamma_{1}, a^{\prime}\right)$ and $T_{Y} F\left(\gamma, a_{\sigma}^{\prime}\right)=q^{\prime}(\sigma)^{-1} T_{Y} F\left(\gamma, a^{\prime}\right)$ for all $\sigma \in \Sigma_{n^{\prime}}$, where $a_{\sigma}^{\prime}=$ $\left(i_{\sigma(1)}, \ldots, i_{\sigma\left(n^{\prime}\right)}\right)$ for each $a^{\prime}=\left(i_{1}, \ldots, i_{n^{\prime}}\right) \in \tilde{\mathbf{N}}^{n^{\prime}}$. Then for $\operatorname{Diff}^{t}(X):\left\|T_{Y} F\right\|^{2}=$ $\sum_{a^{\prime} \in \tilde{N}^{n^{\prime}}} \int_{\Gamma_{Y}}\left\|T_{Y} F\left(\gamma_{1}, a^{\prime}\right)\right\|_{W^{\prime}}^{2} \mu_{1}^{\prime}\left(d \gamma_{1}\right) \leq \sum_{a^{\prime} \in \tilde{N}^{n^{\prime}}} \int_{\Gamma_{X}}\left\|T_{Y} F\left(\gamma, a^{\prime}\right)\right\|_{W^{\prime}}^{2} \mu_{1}^{\prime}(d \gamma)$ $\leq \int_{\Gamma_{Y}} \int_{\Gamma_{Y}^{c}} \sum_{a^{\prime} \in \tilde{\mathbf{N}}^{n^{\prime}}}\left\|T F\left(\gamma_{1}, \gamma_{2}, a^{\prime}\right)\right\|_{W^{\prime}}^{2} \mu_{1}^{\prime}\left(d \gamma_{1}\right) \mu_{2}^{\prime}\left(d \gamma_{2}\right)=\|T F\|^{2} \leq\|F\|^{2}$ in the case $X=M$, consequently, $\left\|T_{Y}\right\| \leq 1$ and $T_{Y}$ is a contraction too. When $\psi \in$ $\operatorname{Dif} f^{t}(Y)$, then $\sigma(\psi, \gamma)$ is independent of $\gamma_{2}$. Hence $T_{Y} U_{m}^{q}(\psi)=U_{m^{\prime}}^{q^{\prime}}(\psi) T_{Y}$ for each $\psi \in \operatorname{Diff}^{t}(Y)$.

There exists the decomposition of $\Gamma_{X}$ into disjoint union of subsets $B_{X_{k}}^{r} \times$ $\Gamma_{X \backslash X_{k}}$ for $r=0,1,2, \ldots$, where each such subset is invariant relative to $\operatorname{Diff} f^{t}\left(X_{k}\right)$, where $k$ is fixed and $B_{X_{k}}^{r}$ is the set of $r$-point subsets in $X_{k}$, $B_{X_{k}}^{0}:=\{\emptyset\}$ is the singleton. Therefore, $L^{2}\left(\Gamma_{X}, \mu, \mathbf{C}\right) \otimes H=\bigoplus_{r=0}^{\infty}\left(L^{2}\left(B_{X_{k}}^{r} \times\right.\right.$ 
$\left.\Gamma_{X \backslash X_{k}}, \mu_{r}, \mathbf{C}\right) \otimes H$, where $\mu_{k}$ is a restriction of $\mu$ on $B_{X_{k}}^{r} \times \Gamma_{X \backslash X_{k}}$ (see also §2.13). Hence $L^{2}\left(B_{X_{k}}^{r} \times \Gamma_{X \backslash X_{k}}, \mu_{r}, \mathbf{C}\right) \otimes H=\bigoplus_{i}\left(L^{2}\left(B_{X_{k}}^{X_{k}} \times \Gamma_{X \backslash X_{k}}, \mu_{r}, \mathbf{C}\right) \otimes\right.$ $\left.W_{r}^{i} \otimes C_{r}^{i}\right)$, where $W_{r}^{i}$ are spaces in which irreducible pairwise non-equivalent unitary representations $q_{r}^{i}$ of the symmetric group $\Sigma_{r}$ act, $C_{r}^{i}$ denote spaces in which $\Sigma_{r}$ acts trivially. Each term in the direct sum is invariant under $\operatorname{Diff}^{t}\left(X_{k}\right)$ such that from $\psi \in \operatorname{Diff}^{t}\left(X_{k}\right)$ and $\gamma \in B_{X_{k}}^{r} \times \Gamma_{X \backslash X_{k}}$ it follows that $\sigma(\psi, \gamma) \in \Sigma_{r}$.

In view of lemma 2.14 the measure $\mu_{r}$ is equivalent with $\mu_{r}^{\prime} \times \mu^{\prime \prime}{ }_{r}$. Hence there exists the isomorphism $\tau_{r}: L^{2}\left(B_{X_{k}}^{r} \times \Gamma_{X \backslash X_{k}}, \mu_{r}, \mathbf{C}\right) \rightarrow L^{2}\left(B_{X_{k}}^{r}, \mu_{r}^{\prime}, \mathbf{C}\right) \otimes$ $L^{2}\left(\Gamma_{X \backslash X_{k}}, \mu{ }_{r}, \mathbf{C}\right)$ given by the following formula: $\tau_{r} F:=\left[\mu_{r}(d \gamma) /\left(\mu_{r}^{\prime}\left(d \gamma^{\prime}\right) \mu^{\prime \prime}{ }_{r}\left(d \gamma^{\prime \prime}\right)\right)\right]^{1 / 2}$ $F(\gamma)$. Hence there exists isomorphism $\tau_{r}: L^{2}\left(B_{X_{k}}^{r} \times \Gamma_{X \backslash X_{k}}, \mu_{r}, \mathbf{C}\right) \otimes W_{r}^{i} \otimes$ $C_{r}^{i} \rightarrow L^{2}\left(B_{X_{k}}^{r}, \mu_{r}^{\prime}, \mathbf{C}\right) \otimes W_{r}^{i} \otimes L^{2}\left(\Gamma_{X \backslash X_{k}}, \mu^{\prime \prime}, \mathbf{C}\right) \otimes C_{r}^{i}$. Therefore, for finitedimensional manifolds $M$ over $\mathbf{R}$ or the local field $\mathbf{L}$ considered here for $\mu=P_{m}$ there is true the following lemma (for finite-dimensional real $M$ see also lemma $3.2[38]$ ).

3.11. Lemma. Under the isomorphism $\tau_{r}$ the operator $U(\psi):=U_{m}^{q}(\psi)$ for each $\psi \in D$ iff $f^{t}\left(X_{k}\right)$ transforms into $\tau_{r} U(\psi) \tau_{r}^{-1}=U_{r}^{i}(\psi) \otimes I$, where $I$ is the unit operator in $L^{2}\left(\Gamma_{X \backslash X_{k}}, \mu_{r}{ }_{r}, \mathbf{C}\right) \otimes C_{r}^{i}$ and $U_{r}^{i}$ is the operator in the space $L^{2}\left(B_{X_{k}}^{r}, \mu_{r}^{\prime}, W_{r}^{i}\right)$ such that $\left(U_{r}^{i}(\psi) F\right)\left(\gamma^{r}\right)=\rho_{\mu_{r}^{\prime}}^{1 / 2}\left(\psi, \gamma^{r}\right) q_{r}^{i}\left(\sigma_{r}\left(\psi, \gamma^{r}\right)\right) F\left(\psi^{-1} \gamma^{r}\right)$, where $\gamma^{r} \in B_{X_{k}}^{r}$.

II. A unitary representation $Q: \Sigma_{\infty} \rightarrow U\left(H^{q}\right)$ such that $Q(\sigma): \phi(a) \mapsto$ $\phi\left(\sigma^{-1} a\right)$ restricted on $\Sigma_{r}$ splits into the direct sum of subspaces: $H^{q}=$ $\oplus_{i} W_{r}^{i} \otimes C_{r}^{i}$, that is, $Q(\sigma) \phi=\sum_{i}\left\{q_{r}^{i} \otimes i d\right\} \phi_{r, i}, \phi=\sum_{i} \phi_{r, i}$, where $\phi_{r, i} \in$ $W_{r}^{i} \otimes C_{r}^{i}, q_{r}^{i}$ are the ireducible and pairwise distinct representations of $\Sigma_{r}$, $\Sigma_{\infty}:=$ ind $-\lim _{r} \Sigma_{r}$. Since $\Gamma_{Y}=\cup_{r=0}^{\infty} B_{Y}^{r}$ is the disjoint union of $B_{Y}^{r}$, then there are the following orthogonal decompositions: $L^{2}\left(\Gamma_{Y}, \mu_{1}, \mathbf{C}\right)=$ $\bigoplus_{r=0}^{\infty} L^{2}\left(B_{Y}^{r}, \mu_{1}, \mathbf{C}\right)$ and $L^{2}\left(\Gamma_{Y}, \mu_{1}, \mathbf{C}\right) \otimes H^{q}=\bigoplus_{r, i} \phi_{\mu}(r, i)$, where $\phi_{\mu}(r, i):=$ $L^{2}\left(B_{Y}^{r}, \mu_{1}, \mathbf{C}\right) \otimes W_{r}^{i} \otimes C_{r}^{i}$ are invariant subspaces of the representation $\left.U_{m}^{q}\right|_{D i f f^{t}(Y)}$. Therefore, $U_{m}^{q}(\psi)=U_{\mu}^{r, i}(\psi) \otimes i d$ on $\phi_{\mu}(r, i)$, where

$($ II.i $) U_{\mu}^{r, i}(\psi)\left(F \otimes w_{r}^{i}\right)=\rho_{\mu_{1}}^{1 / 2}\left(\psi, \gamma_{1}\right) F\left(\psi^{-1}\left(\gamma_{1}\right)\right) q_{r}^{i}(\sigma(\psi, \gamma)) w_{r}^{i}$ for $F \in$ $L^{2}\left(B_{Y}^{r}, \mu_{1}, \mathbf{C}\right)$ and $w_{r}^{i} \in W_{r}^{i}$. The irreducible unitary representations $U_{\mu}^{r, i}$ and $U_{\mu}^{r^{\prime}, i^{\prime}}$ are equivalent if and only if $i=i^{\prime}$ and $r=r^{\prime}$.

Hence there exists the unique integer $J_{i}$ such that either $T_{Y} \phi_{\mu}(r, i)=0$ or $T_{Y} \phi_{\mu}(r, i) \subset \phi_{\mu^{\prime}}\left(r, J_{i}\right)$ and the representations $q_{r}^{i}$ and $q_{r^{\prime}}^{\prime J_{i}}$ are equivalent, consequently, $J_{i} \neq J_{k}$ for each $i \neq k$.

There exist intertwining operators $\omega_{r, i}: W_{r}^{i} \rightarrow W_{r}^{J_{i}}$ of the representa- 
tions $q_{r}^{i}$ and $q_{r}^{\prime J_{i}}$. We denote by $J_{Y}$ the unitary operator $J_{Y}: L^{2}\left(B_{Y}^{r}, \mu_{1}, \mathbf{C}\right) \rightarrow$ $L^{2}\left(B_{Y}^{r}, \mu_{1}^{\prime}, \mathbf{C}\right)$ given by the following formula: $J_{Y} F\left(\gamma_{1}\right):=\left(\mu_{1}\left(d \gamma_{1}\right) / \mu_{1}^{\prime}\left(d \gamma_{1}\right)\right)^{1 / 2} F\left(\gamma_{1}\right)$. Hence

(II.ii) $U_{\mu^{\prime}}^{r, J_{i}}(\psi) T_{r, i}=T_{r, i} U_{\mu}^{r, i}(\psi)$ for each $\psi \in \operatorname{Diff} f^{t}(Y)$, where $T_{r, i}=$ $J_{Y} \otimes \omega_{r, i}: L^{2}\left(B_{Y}^{r}, \mu_{1}, \mathbf{C}\right) \otimes W_{r}^{i} \rightarrow L^{2}\left(B_{Y}^{r}, \mu_{1}^{\prime}, \mathbf{C}\right) \otimes W_{r}^{\prime J_{i}}$.

III. Using the general fact of the representation theory of topological groups from §III of the proof of theorem 3.1 in [36] we get for each $(r, i)$ : either exists a bounded operator $U_{r, i}: C_{r}^{i} \rightarrow C_{r}^{\prime J_{i}}$ such that $\left.T_{Y}\right|_{\phi_{\mu}(r, i)}=T_{r, i} \otimes U_{r, i}$ or $T_{Y} \phi_{\mu}(r, i)=0$. Hence for $\operatorname{Diff}^{t}(Y)$ there is the following equality:

$\left(\right.$ III.i) $T_{Y}(1 \otimes \phi)\left(\gamma, a^{\prime}\right)=\sum_{r, i}^{\prime} T_{r, i} \otimes U_{r, i}\left(\chi_{B_{Y}^{r}} \otimes \phi_{r, i}\right)\left(\gamma, a^{\prime}\right)=\left(m u_{1}\left(d \gamma_{1}\right) / \mu_{1}^{\prime}\left(d \gamma_{1}\right)\right.$ $\sum_{r, i}^{\prime} \chi_{B_{Y}^{r}}\left(\gamma_{1}\right)\left(\omega_{r, i} \otimes U_{r, i}\right)\left(\phi_{r, i}\right)\left(a^{\prime}\right)$, where $\sum^{\prime}$ is a sum for $(r, i)$ such that $T_{Y} \phi_{\mu}(r, i) \neq$ $0, \phi=\operatorname{sum}_{i} \phi_{r, i}, \phi_{r, i} \in W_{r}^{i} \otimes C_{r}^{i}, \chi_{A}$ is the characteristic function of the subset $A$. Then $\left\|\sum_{r, i}^{\prime} \chi_{B_{Y}^{r}}\left(\gamma_{1}\right)\left(\omega_{r, i} \otimes U_{r, i}\right)\left(\phi_{r, i}\right)\left(a^{\prime}\right)\right\|_{W^{\prime}}^{2} \leq \sum_{r} \chi_{B_{Y}^{r}}\left(\gamma_{1}\right) \sum_{i}\left\|\phi_{r, i}\right\|^{2}=1$.

In the case of $\operatorname{Diff}^{t}(X)$ if $P_{m}$ and $P_{m^{\prime}}$ are mutually singular, then $\lim _{k \rightarrow \infty} T_{X_{k}}(1 \otimes \phi)\left(\gamma, a^{\prime}\right)$ converges to $T(1 \otimes \phi)\left(\gamma, a^{\prime}\right)$ for $P_{m^{\prime}}$ a.e. $\gamma$ due to the martingale convergence theorem, but $\lim _{k \rightarrow \infty}\left(\mu_{1}\left(d \gamma_{1}\right) / \mu_{1}^{\prime}\left(d \gamma_{1}\right)\right)^{1 / 2}\left(\gamma_{1}\right) \sum_{r, i}^{\prime} \chi_{B_{Y}^{r}}\left(\gamma_{1}\right)\left(\omega_{r, i} \otimes\right.$ $\left.U_{r, i}\right)\left(\phi_{r, i}\right)\left(a^{\prime}\right)=0$ for $P_{m}$-a.e. $\gamma$, hence $T(1 \otimes \phi)=0$, which contradicts the assumption of this theorem.

IV. In view of theorem 3.9 representations $U_{m}$ are irreducible for $X=G$ or infinite-dimensional $X=M$ over the field $\mathbf{L}$. It was proved in $\S 3.9$ that

$(I V . i)$ the weak closure of subalgebra generated by the family $\left\{U_{m}(h)\right.$ : $\left.h \in G^{\prime}\right\}$ in the algebra of bounded linear operators $L(\bar{H})$ contains all operators of multiplication on functions from the space $L^{\infty}\left(\tilde{\Gamma}_{X}, P_{m}, \mathbf{C}\right)$, where $\bar{H}:=L^{2}\left(\tilde{\Gamma}_{X}, P_{m}, \mathbf{C}\right)$. If measures $P_{m}$ and $P_{m^{\prime}}$ are singular, then

$(I V . i i)$ either $\sup _{\left(\gamma \in \tilde{\Gamma}_{X}\right)}\left|P_{m^{\prime}}(d \gamma) / P_{m}(d \gamma)\right|=\infty$ or $\sup _{\left(\gamma \in \tilde{\Gamma}_{X}\right)}\left|P_{m}(d \gamma) / P_{m^{\prime}}(d \gamma)\right|=$ $\infty$, where $P_{m^{\prime}}(d \gamma) / P_{m}(d \gamma):=\lim _{\left(P_{m}(B) \rightarrow 0, \infty>P_{m}(B)>0, \gamma \in B\right)} P_{m^{\prime}}(B) / P_{m}(B) \in$ $[0, \infty],[0, \infty]:=([0, \infty) \cup\{\infty\}),[0, \infty):=\{x: x \in \mathbf{R}, 0 \leq x\}, B \in$ $B f\left(\tilde{\Gamma}_{X}\right)$. In view of the existence of the intertwining operator $T$ of $U_{m}$ with $U_{m^{\prime}}$ there exists an isomorphism of Hilbert spaces $\tau: L^{2}\left(\tilde{\Gamma}_{X}, P_{m}, \mathbf{C}\right) \rightarrow$ $L^{2}\left(\tilde{\Gamma}_{X}, P_{m^{\prime}}, \mathbf{C}\right)$, which has a continuous extension to an isomorphism of Banach spaces $\tau: L^{\infty}\left(\tilde{\Gamma}_{X}, P_{m}, \mathbf{C}\right) \rightarrow L^{\infty}\left(\tilde{\Gamma}_{X}, P_{m^{\prime}}, \mathbf{C}\right)$ due to condition $(I V . i)$. On the other hand, in view of condition (IV.ii) there exists a sequence $f_{n} \in L^{2}\left(\tilde{\Gamma}_{X}, P_{m}, \mathbf{C}\right)$ such that $C_{1} a_{n} \leq b_{n} \leq C_{2} a_{n}$ for each $n \in \mathbf{N}$ and $\lim _{n \rightarrow \infty} c_{n}<\infty$ and $\lim _{n \rightarrow \infty} d_{n}=\infty$, where $C_{1}$ and $C_{2}$ are positive constants, $a_{n}:=\left\|f_{n}\right\|_{L^{2}\left(\tilde{\Gamma}_{X}, P_{m}, \mathbf{C}\right)}, b_{n}:=\left\|\tau f_{n}\right\|_{L^{2}\left(\tilde{\Gamma}_{X}, P_{m^{\prime}}, \mathbf{C}\right)}, c_{n}:=\left\|f_{n}\right\|_{L^{\infty}\left(\tilde{\Gamma}_{X}, P_{m}, \mathbf{C}\right)}$, $d_{n}:=\left\|\tau f_{n}\right\|_{L^{\infty}\left(\tilde{\Gamma}_{X}, P_{m^{\prime}}, \mathbf{C}\right)}$, since there are sequences $\left\{y_{n}: 0<y_{n}<\infty, n \in \mathbf{N}\right\}$ 
such that $\sum_{n} y_{n}^{-2}<\infty$, but $\sum_{n} y_{n}^{-1}=\infty$. This means that singularity of $P_{m}$ with $P_{m^{\prime}}$ leads to the contradiction, consequently, $P_{m}$ and $P_{m^{\prime}}$ are equivalent. The cases $(\beta)$ are proved analogously with $\mu$ instead of $P_{m}$ and $G$ instead of $\tilde{\Gamma}_{X}$ due to theorem 3.8 .

3.12. Corollary. $(\alpha)$. If $U_{m}^{q}$ and $U_{m^{\prime}}^{q^{\prime}}$ are equivalent as unitary representations, then $P_{m}$ and $P_{m^{\prime}}$ are equivalent as measures.

$(\beta)$. If $T^{\mu}$ and $T^{\mu^{\prime}}$ are equivalent as unitary representations, then $\mu$ and $\mu^{\prime}$ are equivalent as measures.

3.13. Theorem. $(\alpha)$. If $P_{m}$ and $P_{m^{\prime}}$ are equivalent, $n=n^{\prime}$, unitary representations $q$ and $q^{\prime}$ of $\Sigma_{n}$ and $\Sigma_{n^{\prime}}$ are equivalent (in the case Diff $f^{t}(M$ ) of the real manifold $M$ with the additional condition $\operatorname{dim}_{\mathbf{R}} M>1 ; H^{q}=\{0\}$ and $q=I$ for $X=G$ or for infinite-dimensional manifold $X=M$ over the field $\mathbf{L})$. Then the unitary representations $U_{m}^{q}$ and $U_{m^{\prime}}^{q^{\prime}}$ are equivalent.

$(\beta)$. If $\mu$ and $\mu^{\prime}$ from theorem 3.8 are equivalent quasi-invaraint measures on $G$ relative to $G^{\prime}$, then the regular unitary representations $T^{\mu}$ and $T^{\mu^{\prime}}$ are equivalent.

Proof. The cases $(\alpha)$ for $X=M$ infinite-dimensional over the field $\mathbf{L}$ or $X=G$ and $(\beta)$ follow from the fact that $\tau: L^{2}(Z, \mu, \mathbf{C}) \rightarrow L^{2}\left(Z, \mu^{\prime}, \mathbf{C}\right)$ given by the following formula $(\tau f)(x)=\left(\mu(d x) / \mu^{\prime}(d x)\right)^{1 / 2} f(x)$ is the linear topological isomorphism and the intertwining operator of two regular representations in these Hilbert spaces, where either $Z=\tilde{\Gamma}_{X}$ with $\mu=P_{m}$ or $Z=G$ with a quasi-invariant measure $\mu$ relative to $G^{\prime}$ respectively.

It remains only to consider the case of the non-Archimedean manifold $X=M$ with $\operatorname{dim}_{\mathbf{L}} M<\infty$, since the case of real $M$ was proved in $\S 4$ [38]. The measure $m$ on $M$ is supposed to be the restriction of the Haar measure from $\mathbf{L}^{\mathbf{n}}$ on $M$ (see $\left.\$ 2.9\right)$. Let $\operatorname{Diff}^{t}(X, m)$ be a subgroup of $G^{\prime}=\operatorname{Diff} f^{t}(X)$ consisting of diffeomorphisms $\psi$, for which $\rho_{m}(\psi, x)=1$ for each $x \in M$, where $1 \leq t \leq \infty$. In the case of $X=M=\mathbf{R}$ we have $\operatorname{Diff}^{t}(X, m)=\{e\}$, but in the non-Archimedean case each $\psi \in G^{\prime}$ with $\sup _{x \in M}\left|\psi^{\prime}(x)-I\right|<1$ belongs to $\operatorname{Dif} f^{t}(X, m)$. For example, if a countable family of disjoint balls $B_{j}:=B\left(\mathbf{L}^{\mathbf{n}}, x_{j}, r\right)$ with $j \in \Upsilon \subset \mathbf{N}$ of radius $0<r<\infty$ is contained in $M$ and if $\psi \in G^{\prime}$ is such that $\psi\left(B_{j}\right)=B_{\zeta(j)}$ for each $j \in \Upsilon,\left.\psi\right|_{B_{j}}\left(x_{j}+z\right)=$ $x_{\zeta(j)}+\phi_{j}(z)$ for each $z \in B\left(\mathbf{L}^{\mathbf{n}}, 0, r\right),\left.\psi\right|_{\left(M \backslash \bigcup_{j} B_{j}\right)}=i d, \phi_{j}: B\left(\mathbf{L}^{\mathbf{n}}, 0, r\right) \rightarrow$ $B\left(\mathbf{L}^{\mathbf{n}}, 0, r\right)$ are diffeomorphisms with $\sup _{\left(x \in B\left(\mathbf{L}^{\mathbf{n}}, 0, r\right)\right)}\left|\phi_{j}^{\prime}(x)-I\right|<1$ for each $j \in \mathbf{N}$, where $\zeta: \Upsilon \rightarrow \Upsilon$ is a bijection, then $\psi \in \operatorname{Diff}(M, m)$, since $B\left(\mathbf{L}^{\mathbf{n}}, 0, r\right)$ are clopen in $\mathbf{L}^{\mathbf{n}}$ and the valuation group $\left\{|x|_{\mathbf{L}}: 0 \neq x \in \mathbf{L}\right\}$ 
is discrete in $(0, \infty)$. Therefore, in the non-Archimedean case there is not any restriction on $\operatorname{dim}_{\mathbf{L}} M$ from below. If $A \subset \Gamma_{X}$ is invariant by $\bmod \left(P_{m}\right)$ subset of $\Gamma_{X}$ and $P_{m}(A)>0$, then $P_{m}(A)=1$. Indeed, if $P_{m}\left(\Gamma_{X} \backslash A\right)>0$, then there exists $\psi \in \operatorname{Diff}^{t}(X, m)$ such that $P_{m}\left(\left(\Gamma_{X} \backslash A\right) \cap \psi(A)\right)>0$, since $m$ is quasi-invariant relative to $G^{\prime}$ with the continuous quasi-invariant factor $\rho_{m}(h, x)$ by $(h, x) \in G^{\prime} \times \Gamma_{X}$ and $P_{m}((h A) \cap B)$ is the continuous function by $h \in G^{\prime}$ for each $A$ and $B \in A f\left(\Gamma_{X}, P_{m}\right)$, where $A f\left(\Gamma_{X}, P_{m}\right)$ denotes the completion of the Borel $\sigma$-field $B f\left(\Gamma_{X}\right)$ by the ergodic measure $P_{m}$. In view of the invariance of $A$ we have $P_{m}\left(\left(\Gamma_{X} \backslash A\right) \cap A\right)>0$, which is a contradiction, hence $P_{m}\left(\Gamma_{X} \backslash A\right)=0$.

The restriction of the regular unitary representation $\left.U_{m}\right|_{D_{i f f^{t}(X, m)}}$ is given by the following formula:

$\left(U_{m}(\psi) f\right)(\gamma)=f\left(\psi^{-1} \gamma\right)$ for each $\psi \in \operatorname{Diff} f^{t}(X, m)$. Then $f_{0}(\gamma)=1$ for each $\gamma \in \Gamma_{X}$ is the unique vector in $L^{2}\left(\Gamma_{X}, P_{m}, \mathbf{C}\right)$ such that $\mathbf{C} f_{0}$ is invariant relative to $U_{m}(\psi)$ for each $\psi \in D i f f^{t}(M, m)$. The Poisson measure $P_{m}$ can be considered with a parameter $\lambda>0$, that is with $\lambda m$ instead of $m$. Let $u_{m}(\psi)$ be a spherical function given by the following formula: $u_{m}(\psi)=\left(U_{m}(\psi) f_{0}, f_{0}\right)$, where $(*, *)$ denotes the scalar product in $L^{2}\left(\Gamma_{X}, P_{m}, \mathbf{C}\right)$. Then $u_{m}(\psi)=\exp \left(\int_{X}\left(\rho_{m}^{1 / 2}(\psi, x)-1\right) m(d x)\right)$, since $u_{m}(\psi)=$ $\int_{\Gamma_{X}}\left(\prod_{x \in \gamma} \rho^{1 / 2}(\psi, x)\right) P_{m}(d \gamma)$ and for $\operatorname{supp}(\psi) \subset Y$ with $m(Y)<\infty$ we have $u_{m}(\psi)=\left.\sum_{n=0}^{\infty} \int_{B_{Y}^{n}}\left(\prod_{x \in \gamma} \rho^{1 / 2}(\psi, x)\right) P_{m}\right|_{B_{Y}^{n}}(d \gamma)$. Therefore, we get the following theorem.

3.14. Theorem. $(\alpha)$. The representations $U_{\lambda_{1} m}$ and $U_{\lambda_{2} m}$ of Diff $f^{t}(M)$ (with the restriction $\operatorname{dim}_{\mathbf{R}} M>1$ for $M$ over $\mathbf{R}$ and $0<\operatorname{dim}_{\mathbf{L}} M$ in the non-Archimedean case) for $\lambda_{1} \neq \lambda_{2}$ are inequivalent.

$(\beta)$. The representations $U_{\lambda_{1} m}$ and $U_{\lambda_{2} m}$ of $G^{\prime}$ in $L^{2}\left(\tilde{\Gamma}_{G}, P_{\lambda_{j} m}, \mathbf{C}\right)$ with $j=1,2$ respectively are inequivalent for $\lambda_{1} \neq \lambda_{2}$.

Proof. $(\alpha)$. For $\operatorname{dim}_{\mathbf{L}} M<\infty$ this follows from the fact $u_{\lambda_{1} m} \neq u_{\lambda_{2} m}$.

$(\beta)$. In view of the Kakutani theorem [5] two Poisson measures $P_{\lambda_{1} m}$ and $P_{\lambda_{2} m}$ are singular, hence by theorem 3.10 representations $U_{\lambda_{1} m}$ and $U_{\lambda_{2} m}$ are inequivalent.

3.15. Note. By the given above representations it is possible to produce new with the help of the following construction. Let $G^{\prime}$ be a group acting from the left on a $C^{\infty}$-manifold $Y$ (or analytic with disjoint charts in the non-Archimedean case over the local field $\mathbf{L}$ ) such that on $Y$ is given a $\sigma$ additive $\sigma$-finite quasi-invariant non-negative measure $\mu$ with a continuous 
quasi-invariant factor $\rho_{\mu}(\psi, y)$ by $(\psi, y) \in G^{\prime} \times Y$. In the real case let us consider a space $\mathrm{F}(Y)$ of generalised functions on $Y$. For example, if there is a unitary regular representation $T$ of $G^{\prime}$ in $L^{2}(Y, \mu, \mathbf{C})$, then $\mathrm{F}(Y)$ is a space of continuous linear functionals on $L^{2}(Y, \mu, \mathbf{C})$, hence $\mathrm{F}(Y)$ is isomorphic with $L^{2}(Y, \mu, \mathbf{C})$. Let $\nu$ be a measure on $\mathrm{F}(Y)$ given with the help of its characteristic function $\int_{\mathrm{F}(Y)} \exp (i<F, f>) \nu(d F)=\exp \left(-\|f\|^{2} / 2\right)$, where $\|*\|$ is a norm in $L^{2}(Y, \mu, \mathbf{C})$. Such $\nu$ is called the standard Gaussian measure in $\mathrm{F}(Y)$. Then a new representation $\tilde{U}:=E X P_{\beta} T$ is given by the following formula:

$(\tilde{U}(\psi) \Phi)(F):=\exp (i<F, \beta(\psi)>) \Phi\left(T^{*}(\psi) F\right)$, where $<T^{*}(\psi) F, f>=<$ $F, T(\psi) f>,\langle F, f>$ is a value of a functional $F \in \mathrm{F}(Y)$ on a function $f \in L^{2}(Y, \mu, \mathbf{C}), \beta$ is a 1 -cocycle such that $[\beta(\psi)](y):=\rho_{\mu}^{1 / 2}(\psi, y)-1, T(\psi):=$ $\rho^{1 / 2}(\psi, y) f\left(\psi^{-1} y\right)$ for each $\psi \in G^{\prime}, \Phi \in L^{2}(\mathbf{F}, \nu, \mathbf{C}), T^{*}(\psi)=T^{-1}(\psi)=$ $T\left(\psi^{-1}\right)$. If to substitute $\beta$ on $s \beta$, where $s \in \mathbf{R}$, then it produces the oneparameter family $\tilde{U}_{s}:=E X P_{s \beta} T$. There is an equality $\lim _{\psi \rightarrow e} \beta(\psi, y)=0$ for each $y \in Y$. When $Y=G$ or $Y=\tilde{\Gamma}_{X}$ and $\mu$ is as in theorems 3.8 or 3.9, then for $s \neq 0$ representation $\tilde{U}_{s}$ is irreducible as follows from the proof of theorems $3.8,3.9$, since the linear span of non-linear functionals $\exp (i<F, \beta(\psi)>)$ is dense in $L^{2}(\mathrm{~F}(Y), \nu, \mathbf{C})$ (see also $\S 4$ in 38 ). For $X=M$ with $\operatorname{dim}_{\mathbf{L}} M<\infty$ and $s \neq 0$ the representations $\tilde{U}_{s}$ and $U_{s^{2} \mu}$ in $L^{2}\left(\Gamma_{M}, P_{\mu}, \mathbf{C}\right)$ are equivalent, since $\left(\tilde{U}_{s} \psi \Phi_{0}, \Phi_{0}\right)=\exp \left(\int_{M}\left(\rho^{1 / 2}(\psi, x)-1\right) \mu(d x)\right)=u_{s^{2}}(\psi)$, where $\Phi_{0}(F)=1$ for each $F \in \mathrm{F}\left(\Gamma_{M}\right), \Phi_{0} \in L^{2}\left(\mathrm{~F}\left(\Gamma_{M}\right), \nu, \mathbf{C}\right)$.

3.16. Note.It follows from [5, 28, 37], that on $Z$ there are infinite families of orthogonal measures, restrictions of which on $U$ are quasi-invariant relative to $G^{\prime}$ and have continuous quasi-invariance factor on $G^{\prime} \times X$, where either $X=M$ or $X=G$ respectively, $W^{\prime} U \subset V, W^{\prime}$ is open in $G^{\prime}$ and $U$ is open in $Z$. Due to the general procedures of construction of measures on $X$ outlined above on infinite-dimensional $M$ over the corresponding field or on $G$ there are infinite families of orthogonal and as well singular measures, since measures on these infinite-dimensional manifolds $M$ and $G$ are induced from the corresponding Banach spaces $Z$ due to the local diffeomorphisms $A: W \rightarrow V$, where $W$ is open in $M$ or $G$ and $V$ is open in $Z$. Therefore, the last two theorems show that there exists an infinite family of non-equivalent unitary representations of $G^{\prime}$ for $X=G$ and also for $G^{\prime}=\operatorname{Diff}(M)$ for the infinite-dimensional manifold $M$ over the corresponding field, since in these cases on $G$ and $M$ there exist infinite families of orhtogonal measures. The 
unitary group $U\left(l_{2}\right)$ of the standard Hilbert space $l_{2}$ over $\mathbf{C}$ has the topological density c, when $U\left(l_{2}\right)$ is in its standard topolgy induced by the operator norm in the space of linear bounded operators $L\left(l_{2}\right)$ on $l_{2}$, since $l_{2}^{\mathbf{N}}$ in the box topology has a density $\aleph_{0}^{\aleph_{0}}=\mathrm{c}$. When $U\left(l_{2}\right)$ is considered as a topological space in its strong topology [9], then its topological density is $\aleph_{0}$, since $l_{2}^{\mathbf{N}}$ in the product Tychonoff topology has density $\aleph_{0}$ [7]. Therefore, the cardinality of distinct unitary strongly continuous representations $T: G^{\prime} \rightarrow U\left(l_{2}\right)$ for topological group with density $\aleph_{0}$ do not exceed $c$, since $c^{\aleph_{0}}=c[$ [7]. This is important difference of the theory of such non-locally compact topological groups with the theory of compact groups. In the latter case all irreducible unitary representations arise as irreducible components of the regular representation associated with the Haar measure, but for the considered here cases of groups this is not true, since there are infinite families of non-equivalent unitary representations on such groups. There are considered $M$ and $G$ and $\tilde{\Gamma}_{X}$ with countable bases of topology and real-valued measures. The family $\Psi$ of distinct $\sigma$-additive Borel measures on these spaces have the cardinality $\operatorname{card}(\Psi)=\operatorname{card}\left(\mathbf{R}^{\mathbf{N}}\right)=\operatorname{card}(\mathbf{R})=$ : c. In view of theorems 3.10, 3.13, 3.14 and the criteria of orthogonality and singularity of measures on infinitedimensional spaces (using weak distributions, product measures, Kakutani theorem and its non-Archimedean analog [5, 28] and the construction of measures on $G$ or $\tilde{\Gamma}_{X}$ with the help of local diffeomorphisms of open subsets in these spaces and neigbourhoods of zero in the corresponding Banach spaces as in $\S 2.9)$ there exist families $\Psi_{s}$ of singular and $\Psi_{o}$ of orthogonal measures such that $\mathrm{c} \leq \operatorname{card}\left(\Psi_{o}\right) \leq \operatorname{card}\left(\Psi_{s}\right) \leq \operatorname{card}(\Psi)=\mathrm{c}$, hence there are c non-equivalent unitary representations $U_{m}$ of $G^{\prime}$ in $L^{2}\left(\tilde{\Gamma}_{X}, P_{m}, \mathbf{C}\right)$ and also c inequivalent unitary representations of $G^{\prime}$ in $L^{2}(G, \mu, \mathbf{C})$, which were considered above, since $\operatorname{card}\left(\Psi_{s}\right)=\mathrm{c}$.

3.17. Theorem. There exist Abelian non-locally compact Banach-Lie groups $G$ with quasi-invariant measures $\mu$ on $G$ relative to dense subgroups $G^{\prime}$ such that the associated regular unitary representations $T^{\mu}$ of $G^{\prime}$ are irreducible, each one-parameter subgroup $S$ of $G$ is compact and a projection of $\mu$ on each one-parameter subgroup $S$ is equivalent with the Haar measure on $S$.

Proof. Let $l_{2, b}$ be a Hilbert space over $\mathbf{R}$ of elements $x=\left(x_{j}: j \in\right.$ $\left.\mathbf{N}, x_{j} \in \mathbf{R}\right)$ such that $\|x\|_{l_{2, b}}^{2}:=\sum_{j}\left|x_{j} j^{b}\right|^{2}<\infty$. In particular $l_{2}=l_{2,0}$. These spaces have standard orthonormal bases $e_{k}:=(0, \ldots, 0,1,0, \ldots)$ with 
1 on the $k$-th place, $k \in \mathbf{N}$. For a local field $\mathbf{L}$ let $c_{0, b}$ be a Banach space of elements $x=\left(x_{j}: j \in \mathbf{N}, x_{j} \in \mathbf{L}\right)$ such that $\|x\|:=\max _{j}\left|x_{j}\right|_{\mathbf{L}} p^{j b}<\infty$ and $\lim _{j \rightarrow \infty}\left|x_{j}\right| p^{j b}=0$, where $p$ is a prime number such that $\mathbf{L}$ is a finite algebraic extension of the field of $p$-adic numbers $\mathbf{Q}_{\mathbf{p}}$. In particular $c_{0,0}=c_{0}$. If $b>1$ (with $p^{-b} \in \Gamma_{\mathbf{L}}^{\prime}$ in the non-Archimedean case) then the embeddings $J_{b}: l_{2, b} \hookrightarrow l_{2}$ and $S_{b}: c_{0, b} \hookrightarrow c_{0}$ are of trace class: $J_{b} e_{k}=a_{k} e_{k}$ with $a_{k}=k^{-b} \in \mathbf{R}$ and $\sum_{k}\left|a_{k}\right|<\infty, S_{b} e_{k}=v_{k} e_{k}$ with $v_{k} \in \mathbf{L}$ and $\sum_{k}\left|v_{k}\right|<\infty$, where $\left|v_{k}\right|=p^{-j b}$. On $l_{2}$ and $c_{0}$ there exist a Gaussian measure $\lambda$ and a nonArchimedean analog $\eta$ of a Gaussian measure quasi-invariant relative to $l_{2, b}$ and $c_{0, b}$ respectively such that their projections $\lambda_{k}$ and $\eta_{k}$ on one-dimensional subspaces $\mathbf{R} e_{k}$ and $\mathbf{L} e_{k}$ are the following: $\lambda_{k}\left(d x_{k}\right)=C_{k} \exp \left(-x_{k}^{2} s_{k}^{2}\right) w\left(d x_{k}\right)$ and $\eta_{k}\left(d y_{k}\right)=F_{k} \exp \left(-\left|y_{k}\right|^{2} p^{2 k}\right) v\left(d y_{k}\right)$, where $w$ and $v$ are the Lebesgue and the Haar measures on $\mathbf{R}$ and $\mathbf{L}$ respectively such that $w([0,1])=1$, $v(B(\mathbf{L}, 0,1))=1, s_{k}=k^{b^{\prime}}$ for each $k \in \mathbf{N}$ with $1<b^{\prime}<b, C_{k}>0$, $F_{k}>0, \lambda_{k}(\mathbf{R})=1, \eta_{k}(\mathbf{L})=1$ (see [5, 28]). Consider an additive discrete subgroup $E$ of $l_{2, b}$ consisting of elements $x \in l_{2, b}$ such that $x_{j}=n_{j} e_{j}$ for each $j \in \mathbf{N}$, where $n_{j} \in \mathbf{Z}$. Then $l_{2, b} / E=: H_{b}$ and $l_{2} / E=: H$ are the additive groups. The measures $\lambda$ and $\eta$ induce measures $\mu$ on $H$ and $\nu$ on $B\left(c_{0}, 0,1\right):=\left\{x \in c_{0}:\|x\| \leq 1\right\}=: B$. Then $\mu$ is quasi-invariant relative to $H_{b}$ and $\nu$ is quasi-invariant relative to $B\left(c_{0, b}, 0,1\right)$ with continuous quasiinvariance factors such that $\mu(H)=1$ and $\nu(B)>0$.

Let $L_{n}:=s p_{\mathbf{R}}\left(e_{1}, \ldots, e_{n}\right)$ and $E_{n}:=L_{n} \cap E$, so the latter is a discrete subgroup of $L_{n}$ and $L_{n} / E_{n}=: V_{n}$ is a closed subgroup of $H_{b}$. Hence a projection $\pi_{n}: l_{2, b} \rightarrow L_{n}$, which has a continuous extension $\pi_{n}: l_{2} \rightarrow L_{n}$ induces a quotient mapping $\bar{\pi}_{n}: H_{b} \rightarrow V_{n}$ with a continuous extension $\bar{\pi}_{n}: H \rightarrow V_{n}$ for each $n \in \mathbf{N}$. Therefore, the measure $\mu$ on $H$ induces a measure $\mu_{n}$ on $V_{n}$ such that $\mu_{n}(A):=\mu\left(\bar{\pi}_{n}^{-1}(A)\right)$ for each $A \in B f\left(V_{n}\right)$. In view of the equality $\lim _{n \rightarrow \infty} \rho_{\mu_{n}}\left(\bar{\pi}_{n}(\psi), \bar{\pi}_{n}(x)\right)=\rho_{\mu}(\psi, x)$ for each $\psi \in H_{b}$ and $x \in H$ it follows that $\rho_{\mu}(\psi, x)=\lim _{n \rightarrow \infty}\left(\sum_{z \in E_{n}} \exp \left\{\sum_{l=1}^{n}\left[2\left(\psi+z, e_{l}\right)\left(x, e_{l}\right)-\left(\psi+z, e_{l}\right)^{2}\right] s_{l}^{2}\right\}\right)$ $\left(\sum_{z \in E_{n}} \exp \left\{\sum_{l=1}^{n}\left[2\left(z, e_{l}\right)\left(x, e_{l}\right)-\left(z, e_{l}\right)^{2}\right] s_{l}^{2}\right\}\right)^{-1}$, since $\left(\pi_{n}(x), e_{l}\right)=\left(x, e_{l}\right)$ for each $x \in L_{n}$ with $n \geq l$. The Hilbert space $L^{2}(H, \mu, \mathbf{C})$ is isomorphic with a subspace $\left\{f: f \in L^{2}\left(l_{2}, \lambda, \mathbf{C}\right) ; f(x+z)=f(x) \lambda\right.$-a.e. for each $\left.z \in E\right\}$. Since $s p_{\mathbf{C}}\left\{\rho_{\lambda}^{1 / 2}(\psi, x)=: \phi(x) \mid \psi \in l_{2, b}\right\}$ is dense in $L^{2}\left(l_{2}, \lambda, \mathbf{C}\right)$, then $s p_{\mathbf{C}}\left\{\rho_{\mu}^{1 / 2}=\right.$ : $\left.\phi(x) \mid \psi \in H_{b}\right\}$ is dense in $L^{2}(H, \mu, \mathbf{C})$. Repeating the proof of theorem 3.8 for these groups we get that their regular unitary representations are irreducible. That is representations $T$ of $l_{2, b}, H_{b}, c_{0, b}$ and $B\left(c_{0, b}, 0,1\right)$ in the 
corresponding spaces $L^{2}\left(l_{2}, \lambda, \mathbf{C}\right), L^{2}(H, \mu, \mathbf{C}), L^{2}\left(c_{0}, \eta, \mathbf{C}\right)$ and $L^{2}(B, \nu, \mathbf{C})$ (the first case was also considered more generally for additive groups of locally convex spaces in [1, 10]). These groups are Banach-Lie and Abelian, moreover, each one-parameter subgroup of $H_{b}$ and of $B\left(c_{0, b}, 0,1\right)$ over $\mathbf{R}$ and $\mathbf{L}$ respectively is compact. The projections of $\mu$ and $\nu$ on one-parameter subgroups are equivalent with the Haar measures on them. Certainly, $H_{b}$ and $B\left(c_{0, b}, 0,1\right)$ are not locally compact, since $T_{e} H_{b}$ and $T_{e} B\left(c_{0, b}, 0,1\right)$ are infinite-dimensional Banach spaces over $\mathbf{R}$ and $\mathbf{L}$ respectively.

3.18. Note. Regular representations $U_{m}$ of the groups $l_{2, b}, H_{b}, c_{0, b}$ or $B\left(c_{0, b}, 0,1\right)$ from the proof of theorem 3.17 in the space $L^{2}\left(\tilde{\Gamma}_{X}, P_{m}, \mathbf{C}\right)$ with $X=l_{2}, H, c_{0}$ or $B$ and $m=\lambda, \mu, \eta$ or $\nu$ respectively are reducible, since $D^{n} L_{h}=0$ for each $n>1$ and $f_{0}$ is not cyclic for $U_{m}$ (see the proof of theorem $3.9)$.

\section{References}

[1] W. Banaszczyk. Additive subgroups of topological vector spaces (Berlin: Springer, 1991).

[2] D. Bao, J. Lafontaine, T. Ratiu. On a non-linear equation related to the geometry of the difeomorphism group. Pacif. J. Mathem. 158 (1993), $223-242$.

[3] N. Bourbaki. Lie groups and algebras (Moscow: Mir, 1976).

[4] N. Bourbaki. Integration. Chapters 1-9 (Moscow: Nauka, 1970 and 1977).

[5] Yu.L. Dalecky, S.V. Fomin. Measures and differential equations in infinite-dimensional space (Kluwer: Dordrecht, The Netherlands, 1991).

[6] D.G. Ebin, J. Marsden. Groups of diffeomorphisms and the motion of incompressible fluid. Ann. Math. 92 (1970), 102-163.

[7] R. Engelking. General topology (Moscow: Mir,1986).

[8] H.Federer. Geometric measure theory(Berlin:Springer-Verlag, 1969). 
[9] J.M.G. Fell, R.S. Doran. Representations of *-algebras, locally compact groups, and Banach *-algebraic bundles (Acad. Pr.: Boston, 1988).

[10] I.M. Gelfand, N.Ya. Vilenkin. Generalized functions. v. 4. Some applications of harmonic analysis. Rigged Hilbert spaces. (Moscow: Nauka, 1961).

[11] E. Hewitt and K.A. Ross. Abstract harmonic analysis. Second edition (Berlin: Springer-Verlag, 1979).

[12] T. Hirai. Irreducible unitary representations of the group of diffeomorphisms of a non-compact manifold. J. Math. Kyoto Univ. 33 (1993), 827-864.

[13] W. Klingenberg. Riemannian geometry (Walter de Gruyter: Berlin, 1982).

[14] A.V. Kosyak. Irreducible Gaussian representations of the group of the interval and circle diffeomorphisms. J. Funct. Anal. 125(1994), 493-547.

[15] H.-H. Kuo. Gaussian measures in Banach spaces (Springer, Berlin, 1975).

[16] S.V. Ludkovsky. Measurability of representations of infinite-dimensional groups. 51 (1996), 205-206 (N 3).

[17] S.V. Ludkovsky. Measures on groups of diffeomorphisms of nonArchimedean Banach manifolds, Usp. Mat. Nauk. 51(1996), 169-170 (N 2).

[18] S.V. Ludkovsky. Measures on groups of diffeomorphisms of nonArchimedean manifolds, representations of groups and their applications. Theoret. i Mathem. Phys., 1999.

[19] S.V. Ludkovsky. Quasi-invariant measures on non-Archimedean semigroups of loops. Usp. Mat. Nauk, 53 (1998), 203-204 (N 3).

[20] S.V. Ludkovsky. Irreducible unitary representations of non-Archimedean groups of diffeomorphisms. Southeast Asian Bulletin of Mathematics (Hong Kong). 22 (1998), 419-436. 
[21] S.V. Ludkovsky. Irreducible unitary representations of a diffeomorphisms group of an infinite-dimensional real manifold. Rendiconti dell'Istituto di Matematica dell'Università di Trieste. Nuova Serie, (in English) 29 (1998), 22 pages.

[22] S.V. Ludkovsky. Embedding of a non-Archimedean Banach manifold into the corresponding Banach space. Usp. Mat. Nauk., 53 (1998), N 5.

[23] S.V. Ludkovsky. Gaussian quasi-invariant measures on loop groups and semigroups of real manifolds and their representations. IHES, Bures-surYvette, France, preprint IHES/M/97/95.

[24] S.V. Ludkovsky. Quasi-invariant measures on non-Archimedean groups and semigroups of loops and paths, their representations. IHES/M/98/36.

[25] S.V. Ludkovsky. Quasi-invariant measures on groups of diffeomorphisms of Schwarz class of smoothness for real manifolds. IHES/M/97/96.

[26] S.V. Ludkovsky. Representations and structure of groups of diffeomorphisms of non-Archimedean Banach manifolds. I, II. Intern. Centre for Theoret. Phys. Trieste, Italy. Preprints N IC/96/180,181, September 1996 (http://www.ictp.trieste.it).

[27] S.V. Ludkovsky. Quasi-invariant measures on a group of diffeomorphisms of an infinite-dimensional Hilbert manifold and its representations. ICTP. IC/96/202, October 1996.

[28] S.V. Ludkovsky. Quasi-invariant and pseudo-differentiable measures on a non-Archimedean Banach space. ICTP. IC/96/210, October 1996.

[29] S.V. Ludkovsky. Quasi-invariant measures on a non-Archimedean group of diffeomorphisms and on a Banach manifold. ICTP. IC/96/215, October, 1996.

[30] S.V. Ludkovsky. Quasi-invariant measures on groups of diffeomorphisms of real Banach manifolds. ICTP. IC/96/218, October, 1996.

[31] M.A. Naimark. Normed rings (Moscow: Nauka, 1968). 
[32] L. Narici, E. Beckenstein. Topological vector spaces. (Marcel Dekker Inc.: New York, 1985).

[33] Yu.A. Neretin. Representations of the Virasoro algebra and affine algebras. in: Itogi Nauki i Tech. Ser. Sovr. Probl. Math. Fund. Napravl(Moscow: Nauka) 22(1988), 163-230.

[34] A.C.M. van Rooij. Non-Archimedean functional analysis (Marcel Dekker Inc.: New York, 1978).

[35] E.T. Shavgulidze. About one measure quasi-invariant relative to an action of a diffeomorphisms group of a finite-dimensional manifold. Dokl. Akad. Nauk SSSR. 303(1988), 811-814.

[36] H. Shimomura. Poisson measures on the configuration space and unitary representations of the group of diffeomorphisms. J. Math. Kyoto Univ. 34 (1994), 599-614.

[37] A.V. Skorohod. Integration in the Hilbert space (Moscow: Nauka, 1975).

[38] A.M. Vershik, I.M. Gelfand, M.I. Graev. Representations of the group of diffeomorphisms. Usp. Mat. Nauk. 30 (1975), 3-50. 